\title{
QUASI-RANDOM SET SYSTEMS
}

\author{
F. R. K. CHUNG AND R. L. GRAHAM
}

\section{INTRODUCTION}

There are many properties of mathematical objects that satisfy what is sometimes called a $0-1$ law, in the following sense. Under some natural probability measure on the set of objects, the measure of the subset of objects having the given property is either 0 or 1 . In the latter case we can say that almost all the objects have the property. Familiar examples of this phenomenon are the following: almost all real numbers are transcendental (or normal to every base), almost all integers are composite, almost all continuous real functions are nondifferentiable, etc. It is often the case that the objects under consideration can be partitioned into a countable number of finite classes $C_{n}$, with the probability assigned to an object in $C_{n}$ being just $1 /\left|C_{n}\right|$. In this case, we say that a property $P_{n}$ satisfies a 0-1 law if the fraction of the number of objects in $C_{n}$ that satisfy $P_{n}$ either tends to 0 or tends to 1 as $n \rightarrow \infty$. For example, almost all graphs on $n$ vertices have maximum cliques and maximum independent sets of size at most $2 \log n$, almost all Boolean functions with $n$ variables have circuit complexity $(1+o(1)) 2^{n}$ and almost all binary codes of length $n$ with at most $2^{n R}$ codewords (with $R$ less than the binary symmetric channel capacity $C$ ) have arbitrarily small error probability (a special case of Shannon's coding theorem; see [S48]). One of the first general results of this type was the theorem of Fagin [F76] and Glebskii et al. [GKLT69], which asserts that every property of graphs that can be expressed in first-order logic satisfies a 0-1 law (see [SS88] for recent striking developments in this topic).

One obvious method for finding explicit objects having some property $P_{n}$ shared by almost all objects in $C_{n}$ is simply to select one at random. With. overwhelming probability (tending to 1 as $n \rightarrow \infty$ ), the selected object will have property $P_{n}$. Unfortunately, it may be (and often is) extremely difficult to prove that any particular object does indeed satisfy $P_{n}$.

It is our purpose in this paper to describe a method that can to a certain extent circumvent this difficulty. We will show that, for a variety of families, it is possible to identify a natural hierarchy of equivalence classes of properties, all of which are shared by almost all objects in the family. Any object satisfying

Received by the editors June 19, 1990.

1980 Mathematics Subject Classification (1985 Revision). Primary 05C65, 05 C80. 
some property in one of the equivalence classes must of necessity satisfy all of the properties in that class, even though various properties in a class may appear (at first) to be unrelated to each other. Furthermore it is typically easy to verify at least one of the properties in a class (depending upon how the particular object is given), thereby establishing that all the properties in the class hold.

Our main focus in this paper will be on combinatorial objects known as hypergraphs, which are the natural generalizations of graphs (cf. [Be89]). Extensions of these ideas to other structures will be discussed at the end of the paper.

\section{Roots}

Our investigations have their genesis in a number of threads that began to emerge some 20 years or so ago. These are found in the work of Wilson [Wi72, Wi74] on the theory of block designs, Erdös-Sós [ES82] on RamseyTurán problems for graphs and hypergraphs, Rödl [R86] and Graham-Spencer [GS71] (both on certain universality properties of graphs), and more recently Thomason [T87(a), T87(b), T89], Haviland [H89], and Haviland-Thomason [HT89, HT(a)]. Some of the results in this paper have been discussed in a much weaker (nonquantitative) form in several earlier papers of the authors ([CGW89] with R. M. Wilson, [CG90(a), CG(c)]. Here we are able to give the stronger quantitative versions for all of these (and many others as well) and settle a number of the basic questions previously left unanswered. This uniform strengthening has been possible because of the much greater coherence with which the whole subject can now be viewed. In the final section, we speculate on future developments.

\section{Notation}

In this section we introduce a number of definitions that will be used throughout the paper. More specialized definitions will be given later as needed (cf. [Be89]).

For a (finite) set $V$, and a positive integer $k$, define:

$$
\left(\begin{array}{l}
V \\
k
\end{array}\right):=\{X \subset V|| X \mid=k\}, \quad \text { the family of all } k \text {-element subsets of } V,
$$

$V^{k}:=\left\{\left(v_{1}, \ldots, v_{k}\right) \mid v_{i} \in V\right\}, \quad$ the $k$-fold Cartesian product of $V$ with itself.

As usual, $|X|$ denotes the cardinality of the set $X$.

Definition 3.1. A $k$-uniform hypergraph $H=\left(V, \mu_{H}\right)$ consists of a set of $V$ of vertices of $H$, together with a function $\mu_{H}:\left(\begin{array}{l}V \\ k\end{array}\right) \rightarrow\{1,-1\}$, called the (multiplicative) edge function of $H$. The set $E(H):=\mu_{H}^{-1}(-1)$ is called the edge set of $H$, and any $X \in E(H)$ is called an edge of $H$. Its cardinality is denoted by $e(H)$.

Usually, we just call $H$ a $k$-graph. We ordinarily assume $\mu_{H}$ is 1 if two or more of its arguments are equal. Occasionally we will use the additive edge 
function $\chi_{H}$ of $H$ to represent edges of $H$, which is defined by

$$
\chi_{H}(X)= \begin{cases}1 & \text { if } X \in E(H), \\ 0 & \text { otherwise }\end{cases}
$$

We remark that "ordinary graphs" (e.g., see [Bo79] or [BM76]) correspond to the case $k=2$.

The complement $\bar{H}=\left(V, \mu_{\bar{H}}\right)$ of a $k$-graph $H=\left(V, \mu_{H}\right)$ is defined by setting $\mu_{\bar{H}}=-\mu_{H}$. Thus, every $X \in\left(\begin{array}{l}V \\ k\end{array}\right)$ is an edge of exactly one of $H$ and $\bar{H}$. A $k$-graph $G=\left(W, \mu_{G}\right)$ is called a subgraph of $H=\left(V, \mu_{H}\right)$ if there exists an injective mapping $\lambda: W \rightarrow V$ such that $\mu_{G}(X)=-1 \Rightarrow \mu_{H}(\lambda(X))=-1$. In terms of the restriction $\left.\mu_{H}\right|_{\lambda(W)}$ of $\mu_{H}$ to $\lambda(W)$, the previous condition is just $\mu_{G} \leq\left.\mu_{H}\right|_{\lambda(W)}$. Similarly, $G=\left(W, \mu_{G}\right)$ is called an induced subgraph of $H=\left(V, \mu_{H}\right)$ if $\mu_{G}=\left.\mu_{H}\right|_{\lambda(W)}$. We denote the number of occurrences of $G$ as a subgraph, and induced subgraph, of $H$ by $\#\{G \subset H\}$ and $\#\{G<H\}$, respectively.

Given $X \subset V$, define the restriction $H[X]$ of $H$ to $X$ to be the $k$-graph $\left(X, \mu_{H[X]}\right)$ given by setting $\mu_{H[X]}=\left.\mu_{H}\right|_{X}$. For $v \in V$, define the $v$-projection $H(v)$ of $H$ to be the $(k-1)$-graph $\left(V, \mu_{H(v)}\right)$ given by taking

$$
\mu_{H(v)}(Y)=\mu_{H}(Y \cup\{v\}) \text { for } Y \in\left(\begin{array}{c}
V \backslash\{v\} \\
k-1
\end{array}\right) \text {. }
$$

For two $k$-graphs $H=\left(V, \mu_{H}\right), H^{\prime}=\left(V, \mu_{H^{\prime}}\right)$ define the symmetric difference $H \nabla H^{\prime}=\left(V, \mu_{H \nabla H^{\prime}}\right)$ of $G$ and $H$ by setting $\mu_{H \nabla H^{\prime}}=\mu_{H} \mu_{H^{\prime}}$. More generally, the symmetric difference $\nabla_{i=1}^{m} H_{i}$ of the $k$-graphs $H_{i}=\left(V, \mu_{H_{i}}\right)$ is defined to be the $k$-graph $\left(V, \mu^{\nabla}\right)$ with $\mu^{\nabla}=\prod_{i=1}^{m} \mu_{H_{i}}$. For $u, v \in V$, define the sameness $(k-1)$-graph $H_{u, v}$ to be $\overline{H(u) \nabla H(\nu)}$. Thus, $X$ is an edge of $H_{u, v}$ if and only if either both $X \cup\{u\}$ and $X \cup\{v\}$, or neither $X \cup\{u\}$ nor $X \cup\{v\}$, are edges of $H$.

The product $H \square H^{\prime}$ of two $k$-graphs $H=\left(V, \mu_{H}\right)$ and $H^{\prime}=\left(V^{\prime}, \mu_{H^{\prime}}\right)$ is defined to be the $k$-graph $\left(V \times V^{\prime}, \mu_{H \square H^{\prime}}\right)$ where $\mu_{H \square H^{\prime}}\left(X, X^{\prime}\right):=$ $\mu_{H}(X) \mu_{H^{\prime}}\left(X^{\prime}\right)$ for $X \in\left(\begin{array}{c}V \\ k\end{array}\right), X^{\prime} \in\left(\begin{array}{c}V^{\prime} \\ k\end{array}\right)$. In general, the product $\square_{i=1}^{m} H_{i}$ of the $k$-graphs $H_{i}=\left(V_{i}, \mu_{H_{i}}\right)$ is just the $k$-graph $\left(\prod_{i=1}^{m} V_{i}, \mu^{\square}\right)$ with

$$
\mu^{\square}\left(X_{1}, \ldots, X_{m}\right)=\prod_{i=1}^{m} \mu_{H_{i}}\left(X_{i}\right) \text {. }
$$

Note that $X \subset V$ is an edge of $\nabla_{i=1}^{m} H_{i}$ if and only if it is an edge of an odd number of $H_{i}$. Similarly, $\left(X_{1}, X_{2}, \ldots, X_{m}\right) \in \prod_{i=1}^{m} V_{i}$ is an edge of $\square_{i=1}^{m} H_{i}$ if and only if an odd number of $X_{i}$ 's are edges of their respective $H_{i}$ 's.

For a function $\mu:\left(\begin{array}{l}V \\ k\end{array}\right) \rightarrow\{1,-1\}$, denote by $\bar{\mu}$ the extension $\bar{\mu}: V^{k} \rightarrow$ $\{1,-1\}$ defined by

$\bar{\mu}\left(v_{1}, \ldots, v_{k}\right)= \begin{cases}\mu\left(\left\{v_{1}, \ldots, v_{k}\right\}\right) & \text { if } v_{1}, \ldots, v_{k} \text { are distinct elements of } V, \\ 1 & \text { otherwise }\end{cases}$ 
Finally, we come to the most important definition of the section. Let $H=$ $\left(V, \mu_{H}\right)$ be a $k$-graph and assume $|V|=n$.

Definition. The deviation of a $k$-graph $H$, denoted by dev $H$, is defined by

$$
\operatorname{dev} H:=\frac{1}{n^{2 k}} \sum_{\substack{v_{i}(0), v_{i}(1) \in V \\ 1 \leq i \leq k}} \prod_{\substack{\varepsilon_{j} \in\{0,1\} \\ 1 \leq j \leq k}} \bar{\mu}_{H}\left(v_{1}\left(\varepsilon_{1}\right), \ldots, v_{k}\left(\varepsilon_{k}\right)\right)
$$

Using the abbreviations $\bar{v}=\left(v_{1}(0), v_{1}(1), \ldots, v_{k}(0), v_{k}(1)\right) \in V^{2 k}$, and $\bar{\varepsilon}=$ $\left(\varepsilon_{1}, \ldots, \varepsilon_{k}\right) \in\{0,1\}^{k}$, we can shorten the notation for the deviation of $H$ by writing

$$
\operatorname{dev} H=\frac{1}{n^{2 k}} \sum_{v} \prod_{\bar{\varepsilon}} \bar{\mu}_{H}(\bar{v}(\bar{\varepsilon}))
$$

It will be useful to consider a more general form of deviation, defined as follows.

Definition 4.2. For $0 \leq l \leq k$, define the $l$-deviation of the $k$-graph $H=$ $\left(V, \mu_{H}\right)$ (having $n$ vertices), denoted by $\operatorname{dev}_{l} H$, to be

$$
\operatorname{dev}_{l} H:=\frac{1}{n^{k+l}} \sum_{\substack{v_{i}(0), v_{i}(1) \in V \\ 1 \leq i \leq l}} \sum_{\substack{w_{t} \in V \\ l+1 \leq t \leq k}} \prod_{\substack{\varepsilon_{j} \in\{0,1\} \\ 1 \leq j \leq l}} \bar{\mu}_{H}\left(v_{1}\left(\varepsilon_{1}\right), \ldots, v_{l}\left(\varepsilon_{l}\right), w_{l+1}, \ldots, w_{k}\right) .
$$

As before, this can be abbreviated by

$$
\operatorname{dev}_{l} H:=\frac{1}{n^{k+l}} \sum_{\bar{\nu}} \sum_{\bar{w}} \sum_{\bar{\varepsilon}} \bar{\mu}_{H}(\bar{v}(\bar{\varepsilon}), \bar{w}) .
$$

Note that for a $k$-graph $H, \operatorname{dev}_{k} H$ is just $\operatorname{dev} H$.

For the simplest interesting case, namely $k=2$, the deviation of a 2-graph $H$ has the following interpretation. For four (not necessarily distinct) vertices $a, b, c, d$ of $H$, we say that the sequence $(a, b, c, d)$ is an even 4-cycle if an even number of pairs $\{a, b\},\{b, c\},\{c, d\},\{d, a\}$ are edges of $H$. Otherwise, we say that $(a, b, c, d)$ is an odd 4-cycle. Let $\#(\mathrm{E} 4 \mathrm{C} \subset H)$ and $\#(\mathrm{O} 4 \mathrm{C} \subset H)$ denote the numbers of even and odd 4-cycles in $H$, respectively. Then

$$
\operatorname{dev} H=\frac{1}{n^{4}}\{\#(\mathrm{E} 4 \mathrm{C} \subset H)-\#(\mathrm{O} 4 \mathrm{C} \subset H)\} .
$$

Similarly, for the general case, the deviation of a $k$-graph $H$ can be interpreted as the average difference between the numbers of even and odd "octahedra" in $H$, where an octahedron is a certain $2 k$-vertex $k$-graph having $2^{k}$ edges (see [CG90] for details).

A standard model (e.g., see [Bo85]) for discussing random $k$-graphs on a set $V$ assigns $\mu(X)=1$ or -1 , each with probability $1 / 2$ independently for each $X \in\left(\begin{array}{l}V \\ k\end{array}\right)$. This process actually induces a probability distribution on the set of 
all possible $k$-graphs of $V$. We say that almost all $k$-graphs have some specified property $P$ if the probability that a $k$-graph generated by this process tends to 1 as $|V|=n \rightarrow \infty$. We will usually denote a typical $k$-graph so generated by $H_{1 / 2}^{(k)}(n)$, or $H_{1 / 2}(n)$ if $k$ is understood.

The main thrust of many of the results in this paper is that many of the properties shared by almost all random $k$-graphs are in fact implied by (and imply) the condition that the deviation of a $k$-graph is small. Specifically, the smaller $\operatorname{dev} H$ is, the more $H$ behaves like a random $k$-graph (e.g., see Theorems $6.1,8.1$, and 9.1). In this sense, $\operatorname{dev} H$ is a fundamental invariant of $H$ as far as characterizing its random aspects. For this reason, $k$-graph properties equivalent to the vanishing of $\operatorname{dev} H$ are called quasi-random (cf. [CGW89, CG90(a)]).

\section{BASIC PROPERTIES OF DEVIATION}

In this section we summarize some of the fundamental properties of deviation. Unless specified otherwise, $H=\left(V, \mu_{H}\right)$ will denote a $k$-graph with $|V|=n$.

Fact 4.0. $\operatorname{dev} \bar{H}=\operatorname{dev} H$.

Proof. Immediate from definition of deviation.

Fact 4.1.

$$
0 \leq \operatorname{dev} H \leq 1 .
$$

Proof. The upper bound is an immediate consequence of the definition. For the lower bound we have by $(3.1)$

$$
\begin{aligned}
\operatorname{dev} H & =\frac{1}{n^{2 k}} \sum_{\substack{v_{i}(0), v_{i}(1) \in V \\
1 \leq i \leq k}} \prod_{\substack{\varepsilon_{j} \in\{0,1\} \\
1 \leq j \leq k}} \bar{\mu}_{H}\left(v_{1}\left(\varepsilon_{1}\right), \ldots, v_{k}\left(\varepsilon_{k}\right)\right) \\
& =\frac{1}{n^{2 k}} \sum_{\substack{v_{i}(0), v_{i}(1) \in V \\
1 \leq i \leq k-1}}\left(\sum_{\substack{w \in V \\
\varepsilon_{j} \in\{0,1\} \\
1 \leq j \leq k-1}} \prod_{H}\left(v_{1}\left(\varepsilon_{1}\right), \ldots, v_{k-1}\left(\varepsilon_{k-1}\right), w\right)\right)^{2} \\
& \geq 0 .
\end{aligned}
$$

By keeping track of those terms in $\operatorname{dev} H$ that have a repeated coordinate (causing $\bar{\mu}_{H}$ to take the value 1), we can obtain the following somewhat stronger lower bound for $\operatorname{dev} H$.

\section{Fact 4.2.}

$$
\operatorname{dev} H \geq 1-(1-1 / n)^{k-1} .
$$

In particular, this implies that

$$
\operatorname{dev} H \geq 1 / n \text { for } k \geq 2,
$$

a fact that will be used to help simplify the forms of various inequalities. 


\section{Fact 4.3.}

$$
\operatorname{dev} H=\frac{1}{n^{2}} \sum_{v, v^{\prime} \in V} \operatorname{dev}\left(H(v) \nabla H\left(v^{\prime}\right)\right)
$$

Proof.

$$
\begin{aligned}
& \operatorname{dev}(\left.H(v) \nabla H\left(v^{\prime}\right)\right) \\
&= \frac{1}{n^{2 k-2}} \sum_{\substack{v_{i}(0), v_{i}(1) \in V \\
1 \leq i \leq k-1}} \prod_{\substack{\varepsilon_{j} \in\{0,1\} \\
1 \leq j \leq k-1}} \bar{\mu}_{H(v) \nabla H\left(v^{\prime}\right)}\left(v_{1}\left(\varepsilon_{1}\right), \ldots, v_{k-1}\left(\varepsilon_{k-1}\right)\right) \\
&= \frac{1}{n^{2 k-2}} \sum_{\substack{v_{i}(0), v_{i}(1) \in V \\
1 \leq i \leq k-1}} \prod_{\substack{\varepsilon_{j} \in\{0,1\} \\
1 \leq j \leq k-1}} \bar{\mu}_{H}\left(v_{1}\left(\varepsilon_{1}\right), \ldots, v_{k-1}\left(\varepsilon_{k-1}\right), v\right) \\
& \times \bar{\mu}_{H}\left(v_{1}\left(\varepsilon_{1}\right), \ldots, v_{k-1}\left(\varepsilon_{k-1}\right), v^{\prime}\right) .
\end{aligned}
$$

Thus,

$$
\begin{aligned}
& \frac{1}{n^{2}} \sum_{v, v^{\prime} \in V} \operatorname{dev}\left(H(v) \nabla H\left(v^{\prime}\right)\right) \\
& \quad=\frac{1}{n^{2 k}} \sum_{v, v^{\prime}} \sum_{\substack{v_{i}(0), v_{i}(1) \\
1 \leq i \leq k-1}} \prod_{\substack{\varepsilon_{j} \in\{0,1\} \\
1 \leq j \leq k-1}} \bar{\mu}_{H}\left(v_{1}\left(\varepsilon_{1}\right), \ldots, v\right) \bar{\mu}_{H}\left(v_{1}\left(\varepsilon_{1}\right), \ldots, v^{\prime}\right) \\
& \quad=\frac{1}{n^{2 k}} \sum_{\substack{v_{i}(0), v_{i}(1) \\
1 \leq i \leq k}} \prod_{\substack{\varepsilon_{j} \in\{0,1\} \\
1 \leq j \leq k}} \bar{\mu}_{H}\left(v_{1}\left(\varepsilon_{1}\right), \ldots, v_{k}\left(\varepsilon_{k}\right)\right)=\operatorname{dev} H . \quad
\end{aligned}
$$

Essentially the same proof yields the more general result:

Fact 4.4. For $0 \leq l \leq k$,

$$
\operatorname{dev}_{l} H=\frac{1}{n^{2}} \sum_{v, v^{\prime} \in V} \operatorname{dev}_{l-1}\left(H(v) \nabla H\left(v^{\prime}\right)\right) .
$$

Fact 4.5. For $k$-graphs $H=\left(V, \mu_{H}\right), H^{\prime}=\left(V^{\prime}, \mu_{H^{\prime}}\right)$,

$$
\operatorname{dev} H \square H^{\prime}=\operatorname{dev} H \operatorname{dev} H^{\prime} \text {. }
$$

Proof.

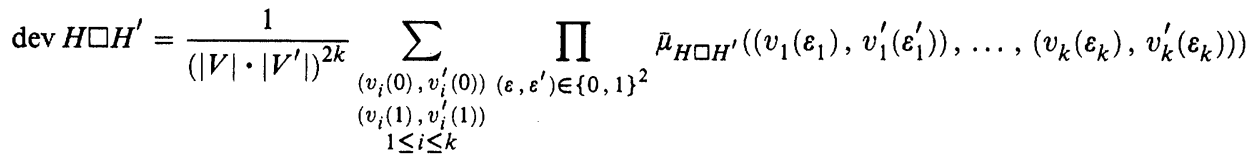

$$
\begin{aligned}
& =\frac{1}{|V|^{2 k}\left|V^{\prime}\right|^{2 k}} \sum_{\substack{v_{i}(0), v_{i}(1) \\
v_{i}^{\prime}(0), v_{i}^{\prime}(1) \\
1 \leq i \leq k}} \prod_{\substack{\left(\varepsilon, \varepsilon^{\prime}\right) \in\{0,1\}^{2} \\
\mu^{2}}} \bar{\mu}_{H}\left(v_{1}\left(\varepsilon_{1}\right), \ldots, v_{k}\left(\varepsilon_{k}\right)\right) \bar{\mu}_{H^{\prime}}\left(v_{1}^{\prime}\left(\varepsilon_{1}^{\prime}\right), \ldots, v_{k}^{\prime}\left(\varepsilon_{k}^{\prime}\right)\right) \\
& =\operatorname{dev} H \operatorname{dev} H^{\prime} .
\end{aligned}
$$

The same proof shows the following more general result. 
Fact 4.6. For $0 \leq l \leq k$,

$$
\operatorname{dev}_{l} H \square H^{\prime}=\operatorname{dev}_{l} H \operatorname{dev}_{l} H^{\prime} .
$$

Of course, Fact 4.6 generalizes to any number of factors:

$$
\operatorname{dev}_{l} \square_{1 \leq i \leq m} H_{i}=\prod_{1 \leq i \leq m} \operatorname{dev}_{l} H_{i} .
$$

Fact 4.7. For $X \subset V$,

$$
\operatorname{dev} H[X] \leq(|V| /|X|)^{2 k} \operatorname{dev} H .
$$

Proof.

$$
\begin{aligned}
& \operatorname{dev} H=\frac{1}{n^{2 k}} \sum_{\substack{v_{i}(0), v_{i}(1) \in V \\
1 \leq i \leq k}} \prod_{\substack{\varepsilon_{j} \in\{0,1\} \\
1 \leq j \leq k}} \bar{\mu}_{H}\left(v_{1}\left(\varepsilon_{1}\right), \ldots, v_{k}\left(\varepsilon_{k}\right)\right) \\
& =\frac{1}{n^{2 k}} \sum_{\substack{v_{i}(0), v_{i}(1) \in V \\
1 \leq i \leq k-1}}\left(\sum_{v \in V} \prod_{\substack{\varepsilon_{j} \in\{0,1\} \\
1 \leq j \leq k-1}} \bar{\mu}_{H}\left(v_{1}\left(\varepsilon_{1}\right), \ldots, v_{k-1}\left(\varepsilon_{k-1}\right), v\right)\right)^{2} \\
& \geq \frac{1}{n^{2 k}} \sum_{\substack{v_{i}(0), v_{i}(1) \in X \\
1 \leq i \leq k-1}}\left(\sum_{v \in V} \prod_{\substack{\varepsilon_{j} \in\{0,1\} \\
1 \leq j \leq k-1}} \bar{\mu}_{H}\left(v_{1}\left(\varepsilon_{1}\right), \ldots, v_{k-1}\left(\varepsilon_{k-1}\right), v\right)\right)^{2} \\
& =\frac{1}{n^{2 k}} \sum_{\substack{v_{k}(0), v_{k}(1) \in V \\
v_{i}(0), v_{i}(1) \in X \\
1 \leq i \leq k-1}} \prod_{\substack{\varepsilon_{j} \in\{0,1\} \\
1 \leq j \leq k}} \bar{\mu}_{H}\left(v_{1}\left(\varepsilon_{1}\right), \ldots, v_{k}\left(\varepsilon_{k}\right)\right) \\
& =\frac{1}{n^{2 k}} \sum_{\substack{v_{k}(0), v_{k}(1) \in V \\
v_{i}(0), v_{1}(1) \in X \\
2 \leq i \leq k-1}}\left(\sum_{\substack{v \in X \\
\varepsilon_{j} \in\{0,1\} \\
2 \leq i \leq k}} \bar{\mu}_{H}\left(v, v_{2}\left(\varepsilon_{2}\right), \ldots, v_{k}\left(\varepsilon_{k}\right)\right)\right)^{2} \\
& \geq \frac{1}{n^{2 k}} \sum_{\substack{v_{i}(0), v_{i}(1) \in X \\
2 \leq i \leq k}}\left(\sum_{v \in X} \prod_{\substack{\varepsilon_{j} \in\{0,1\} \\
2 \leq i \leq k}} \bar{\mu}_{H}\left(v, v_{2}\left(\varepsilon_{2}\right), \ldots, v_{k}\left(\varepsilon_{k}\right)\right)\right)^{2} \\
& =\frac{1}{n^{2 k}} \sum_{\substack{v_{i}(0), v_{i}(1) \in X \\
1 \leq i \leq k}} \prod_{\substack{\varepsilon_{j} \in\{0,1\} \\
1 \leq j \leq k}} \bar{\mu}_{H}\left(v_{1}\left(\varepsilon_{1}\right), \ldots, v_{k}\left(\varepsilon_{k}\right)\right) \\
& =(|X| /|V|)^{2 k} \operatorname{dev} H[X]
\end{aligned}
$$

as required.

Fact 4.7 has a sort of converse, which asserts that if a family of subsets $X_{i}$ of $V$ covers $\left(\begin{array}{c}V \\ 2 k\end{array}\right)$ fairly uniformly then $\operatorname{dev} H$ is close to the average of the 
$\operatorname{dev} H\left[X_{i}\right]$. More precisely:

Fact 4.8. Suppose $X_{1}, \ldots, X_{m} \subset V$ so that

(i) each $Y \in\left(\begin{array}{c}V \\ 2 k\end{array}\right)$ occurs in $(1+o(1)) t$ of the $X_{r}$;

(ii) $\left|X_{r}\right|=(1+o(1)) u, 1 \leq r \leq m$.

Then

$$
\operatorname{dev} H \leq(1+o(1)) \frac{1}{m} \sum_{r=1}^{m} \operatorname{dev} H\left[X_{r}\right]+O\left(\frac{1}{n}\right), \quad n \rightarrow \infty .
$$

Proof. First, observe that the hypotheses imply

$$
\frac{m}{t}=(1+o(1))\left(\begin{array}{c}
n \\
2 k
\end{array}\right) /\left(\begin{array}{c}
u \\
2 k
\end{array}\right) .
$$

Thus, with $n^{\underline{t}}:=n ! /(n-t) !$ we have

$$
\begin{aligned}
\operatorname{dev} H & =\frac{1}{n^{2 k}} \sum_{\substack{v_{i}(0), v_{i}(1) \\
1 \leq i \leq k}} \prod_{\substack{\varepsilon_{j} \\
1 \leq j \leq k}} \bar{\mu}_{H}\left(v_{1}\left(\varepsilon_{1}\right), \ldots, v_{k}\left(\varepsilon_{k}\right)\right) \\
& \leq \frac{1}{n^{2 k}}\left(n^{2 k}-n^{\frac{2 k}{2 k}}+\sum_{\substack{\text { distinct } \\
v_{i}(0), v_{i}(1) \\
1 \leq i \leq k}} \prod_{\substack{\varepsilon_{j} \\
1 \leq j \leq k}} \bar{\mu}_{H}\left(v_{1}\left(\varepsilon_{1}\right), \ldots, v_{k}\left(\varepsilon_{k}\right)\right)\right) \\
& =O\left(\frac{1}{n}\right)+\frac{(1+o(1))}{t n^{2 k}} \sum_{1 \leq r \leq m} \sum_{v_{i}(0), v_{i}(1) \in X_{r}} \sum_{\varepsilon_{j}} \bar{\mu}_{H\left[X_{r}\right]}\left(v_{1}\left(\varepsilon_{1}\right), \ldots, v_{k}\left(\varepsilon_{k}\right)\right) \\
& =O\left(\frac{1}{n}\right)+(1+o(1)) \frac{1}{t}\left(\frac{u}{n}\right)^{2 k} \sum_{1 \leq r \leq m} \operatorname{dev} H\left[X_{r}\right] \\
& =(1+o(1)) \frac{1}{m} \sum_{r=1}^{m} \operatorname{dev} H\left[X_{r}\right]+O\left(\frac{1}{n}\right) \quad \text { as } n \rightarrow \infty .
\end{aligned}
$$

Fact 4.9. For $1 \leq l \leq k$,

$$
\operatorname{dev}_{l} H \geq\left(\operatorname{dev}_{l-1} H\right)^{2} .
$$

Proof.

$$
\begin{aligned}
\operatorname{dev}_{l} H & =\frac{1}{n^{k+l}} \sum_{\substack{v_{i}(0), v_{i}(1) \\
1 \leq i \leq l}} \sum_{\substack{w_{t} \\
l+1 \leq t \leq k}} \prod_{\substack{\varepsilon_{j} \in\{0,1\} \\
1 \leq j \leq l}} \bar{\mu}_{H}\left(x_{1}\left(\varepsilon_{1}\right), \ldots, x_{l}\left(\varepsilon_{l}\right), w_{l+1}, \ldots, w_{k}\right) \\
& =\frac{1}{n^{k+l}} \sum_{\substack{v_{i}(0), v_{i}(1) \\
1 \leq i \leq l-1}} \sum_{\substack{w_{t} \\
l+1 \leq t \leq k}}\left(\sum_{v} \prod_{\substack{\varepsilon_{j} \in\{0,1\} \\
1 \leq j \leq l-1}} \bar{\mu}_{H}\left(x_{1}\left(\varepsilon_{1}\right), \ldots, v, w_{l+1}, \ldots, w_{k}\right)\right)^{2} \\
& \geq \frac{1}{n^{k+l}} \cdot \frac{1}{n^{k+l-2}}\left(\sum_{\substack{v_{i}(0), v_{i}(1) \\
1 \leq i \leq l-1}} \sum_{\substack{w_{t} \\
1 \leq t \leq k}} \prod_{e_{j} \in\{0,1\}} \bar{\mu}_{H}\left(x_{1}\left(\varepsilon_{1}\right), \ldots, x_{l-1}\left(\varepsilon_{l-1}\right), w_{l}, \ldots, w_{k}\right)\right)^{2}
\end{aligned}
$$$$
=\left(\operatorname{dev}_{l-1} H\right)^{2} \cdot \square
$$

by the Cauchy-Schwarz inequality 
By taking $l=k$, we obtain the lower bound of (4.1). Note that when $l=0$,

$$
\operatorname{dev}_{0} H=\frac{1}{n^{k}} \sum_{\substack{w_{j} \in V \\ 1 \leq j \leq k}} \bar{\mu}_{H}\left(w_{1}, \ldots, w_{k}\right)=1-\frac{2 k !}{n^{k}}|E(H)| .
$$

Fact 4.10. For $0 \leq l<k$,

$$
\operatorname{dev}_{l} H=\frac{1}{n} \sum_{v \in V} \operatorname{dev}_{l} H(v)
$$

Proof.

$$
\begin{aligned}
\operatorname{dev}_{l} H & =\frac{1}{n^{k+l}} \sum_{v_{i}(0), v_{i}(1)} \sum_{\substack{w_{t} \\
1 \leq i \leq l}} \prod_{\substack{\varepsilon_{j} \in\{0,1\} \\
l+1 \leq t \leq k \\
1 \leq j \leq l}} \bar{\mu}_{H}\left(x_{1}\left(\varepsilon_{1}\right), \ldots, x_{l}\left(\varepsilon_{l}\right), w_{l+1}, \ldots, w_{k}\right) \\
& =\frac{1}{n} \sum_{w} \frac{1}{n^{k+l-1}} \sum_{\substack{v_{i}(0), v_{i}(1) \\
1 \leq i \leq l}} \sum_{\substack{w_{t} \\
l+1 \leq t \leq k-1}} \prod_{\substack{\varepsilon_{j} \in\{0,1\} \\
1 \leq j \leq l}} \bar{\mu}_{H}\left(x_{1}\left(\varepsilon_{1}\right), \ldots, w_{k-1}, w\right) \\
& =\frac{1}{n} \sum_{w} \frac{1}{n^{k+l-1}} \sum_{\substack{v_{i}(0), v_{i}(1) \\
1 \leq i \leq l}} \sum_{\substack{w_{t} \\
l+1 \leq t \leq k-1}} \prod_{\substack{\varepsilon_{j} \in\{0,1\} \\
1 \leq j \leq l}} \bar{\mu}_{H(w)}\left(x_{1}\left(\varepsilon_{1}\right), \ldots, w_{k-1}\right) \\
& =\frac{1}{n} \sum_{w} \operatorname{dev}_{l} H(w) .
\end{aligned}
$$

Finally, we note the observation (to be used later)

$$
\operatorname{dev} G=1 \Rightarrow \operatorname{dev} G \nabla H=\operatorname{dev} H .
$$

The proof follows at once from the definitions.

\section{SOME PROPERTIES OF RANDOM $k$-GRAPHS}

In this section we describe a variety of properties shared by almost all random $k$-graphs. These will serve as reference points for comparing the corresponding quasi-random versions of these properties in later sections. Proofs of the assertions are not difficult, or are available in the literature, and (with exception of Fact 5.1) are omitted. As usual, $H=H_{1 / 2}^{(k)}(n)$ will denote the random $k$-graph on $n$ vertices as described in $\S 1$.

\section{Fact 5.1.}

$$
\operatorname{Pr}\left[\operatorname{dev} H_{1 / 2}^{(k)}(n)>\frac{\alpha}{n}\right] \leq \frac{1}{\alpha}\left(\begin{array}{c}
2 k \\
2
\end{array}\right) .
$$

Proof. For $\bar{v}=\left(v_{1}(0), v_{1}(1), \ldots, v_{k}(0), v_{k}(1)\right) \in V^{2 k}$, let

$$
Y(\bar{v}):=\prod_{\varepsilon_{1}, \ldots, \varepsilon_{k} \in\{0,1\}} \bar{\mu}_{H}\left(v_{1}\left(\varepsilon_{1}\right), \ldots, v_{k}\left(\varepsilon_{k}\right)\right)
$$


and let $S:=\sum_{v \in V^{2 k}} Y(\bar{v})$. Thus, $S=n^{2 k} \operatorname{dev} H$. Let us call $\bar{v} \in V^{2 k}$ proper if all its components are distinct; otherwise we say it is degenerate. Observe that the number $N$ of degenerate $\bar{v}$ satisfies

$$
N=n^{2 k}-n^{2 k} \leq\left(\begin{array}{c}
2 k \\
2
\end{array}\right) n^{2 k-1} \text {. }
$$

Thus,

$$
\begin{aligned}
E[S] & =E\left[\sum_{v} Y(\bar{v})\right]=\sum_{v} E[Y(\bar{v})] \\
& =\sum_{v \text { degenerate }} E[Y(\bar{v})]+\sum_{v \text { proper }} E[Y(\bar{v})] \\
& \leq\left(\begin{array}{c}
2 k \\
2
\end{array}\right) n^{2 k-1} \cdot 1+0
\end{aligned}
$$

since for proper $\bar{v}$, all the factors in $Y(\bar{v})$ are independent. Writing Chebyshev's inequality as

$$
\operatorname{Pr}[S>\lambda] \leq \frac{E[S]}{\lambda}
$$

then taking $\lambda=\alpha n^{2 k-1}$, we obtain (5.1).

We remark that, with a little more care, it can be shown that for appropriate constants $c_{k}$ and $c>1$,

$$
\operatorname{Pr}\left[\operatorname{dev} H_{1 / 2}^{(k)}(n)>c_{k} / n\right]<c^{-\sqrt{n}} .
$$

Fact 5.2. Let $H(t)$ be an arbitrary fixed $k$ graph on $t$ vertices. Then

$$
\#\left\{H(t)<H_{1 / 2}(n)\right\}=(1+o(1)) \frac{n^{t}}{\left.{ }_{2}^{(} \begin{array}{l}
t \\
k
\end{array}\right)}, \quad n \rightarrow \infty .
$$

Fact 5.3. Let $H:=H_{1 / 2}^{(k)}(n)$. Then almost all $H$ satisfy $($ as $n \rightarrow \infty)$

(i) $|E(H)|=\left(\frac{1}{2}+o(1)\right)\left(\begin{array}{l}n \\ k\end{array}\right)$;

(ii) for almost all vertices $v \in V$ of $H,|E(H(v))|=\left(\frac{1}{2}+o(1)\right)\left(\begin{array}{c}n \\ k-1\end{array}\right)$;

(iii) for all $X \subset V,|E(H[X])|=\frac{1}{2}\left(\begin{array}{c}|X| \\ k\end{array}\right)+o\left(n^{k}\right)$.

Of course, (iii) $\Rightarrow$ (ii) $\Rightarrow$ (i). We will see the relevance of these properties to the $l$-deviation of a $k$-graph later.

The deviation of a $k$-graph is related to a special $k$-graph called a $k$-octahedron, $\mathscr{O}=\left(V, \mu_{\mathscr{O}}\right)$, defined as follows. The vertex set $V$ consists of $2 k$ points $x_{i}(0), x_{i}(1), 1 \leq i \leq k$. The edges of $\mathscr{O}$ consist of all $k$-sets of the form $\left\{x_{1}\left(\varepsilon_{1}\right), \ldots, x_{k}\left(\varepsilon_{k}\right): \varepsilon_{1}, \ldots, \varepsilon_{k}=0\right.$ or 1$\}$ (so that $\mathscr{O}$ has $2^{k}$ edges). A partial octahedron is a subgraph of $\mathscr{O}$ having as edges a subset of the edges of $\mathscr{O}$. More specifically, an even partial octahedron (EPO) has an even number of such edges, while an odd partial octahedron (OPO) has an odd number. We let $\#\{\mathrm{EPO}<H\}$ and $\#\{\mathrm{OPO}<H\}$ denote the numbers of induced EPO's and OPO's, respectively, in $H$. 
In these terms, Fact 5.1 asserts that

$$
\#\left\{\mathrm{EPO}<H_{1 / 2}^{(k)}(n)\right\}-\#\left\{\mathrm{OPO}<H_{1 / 2}^{(k)}(n)\right\}=o\left(\left(\begin{array}{l}
n \\
k
\end{array}\right)\right) .
$$

In the case of ordinary graphs $H=H^{(2)}(n)=\left(V, \chi_{H}\right)$, we define the adjacency matrix $A(H):=\left(\chi_{H}(x, y)\right)_{x, y \in V}$. Since $A(H)$ is real and symmetric, it has real eigenvalues $\lambda_{i}$, which can be labelled so that $\left|\lambda_{1}\right| \geq\left|\lambda_{2}\right| \geq \cdots \geq\left|\lambda_{n}\right|$.

Fact 5.4 [FK81]. Almost all random graphs $H_{1 / 2}(n)$ have $\lambda_{1}=(1+o(1)) n / 2$, $\lambda_{2}=O\left(n^{1 / 2}\right)$.

\section{INDUCED SUBGRAPHS}

As we have noted, for a fixed $k$-graph $G(t)$ on $t$ vertices, almost all random $k$-graphs $H_{1 / 2}(n)$ contain $(1+o(1)) n^{t} / 2^{(}\left(\begin{array}{l}t \\ k\end{array}\right)$ occurrences of $G(t)$ as an induced subgraph. In this section we will show that this property actually holds for any $k$-graph $H(n)$ with small deviation. More precisely, we prove

Theorem 6.1. Assume $G=G(t)$ is a fixed $k$-graph on $t$ vertices where $k \geq 2$. Then for any $H=H(n)$ with $n \geq t^{2}$, we have

$$
\left|\#\{G(t)<H(n)\}-\frac{n^{t}}{2\left(\begin{array}{l}
t \\
k
\end{array}\right)}\right| \leq 5 n^{t}(\operatorname{dev} H(n))^{2^{-k}} .
$$

Proof. Denote the vertex set of $G$ by $V=\left\{v_{1}, \ldots, v_{t}\right\}$. For $1 \leq r \leq t$, define $V_{r}:=\left\{v_{1}, \ldots, v_{r}\right\} \subset V, G(r):=G\left[V_{r}\right]$, and $N_{r}:=\#\{G(r)<H\}$. We will show by induction on $r$ and $k$ that

$$
\left|N_{r}-\frac{n^{\underline{r}}}{2\left(\begin{array}{l}
r \\
k
\end{array}\right)}\right| \leq 5 n^{r}(\operatorname{dev} H)^{2^{-k}} .
$$

First note that for $r<k, N_{r}=n^{\underline{r}}$ and (6.2) holds since

$$
\left|N_{r}-\frac{n^{\underline{r}}}{2^{0}}\right|=0 \leq 5 n^{r}(\operatorname{dev} H)^{2^{-k}} \text {. }
$$

So let us assume for a fixed value of $k \geq 2$ that (6.2) holds for some value of $r$ satisfying $k-1 \leq r<t$. We want to prove that (6.2) also holds for $r+1$.

Denote the vertex set of $H$ by $[n]=\{1,2, \ldots, n\}$. Let $\alpha$ denote $\left(\alpha_{1}, \ldots\right.$, $\alpha_{r}$ ) where the $\alpha_{i}$ are distinct elements of $[n]$, and let $\varepsilon$ denote

$$
\left(\varepsilon\left(e_{1}\right), \ldots, \varepsilon\left(e_{z}\right)\right) \quad \text { with } \varepsilon\left(e_{i}\right) \in\{-1,1\}, 1 \leq i \leq z:=\left(\begin{array}{c}
r \\
k-1
\end{array}\right),
$$

where $e_{1}, \ldots, e_{z}$ denotes an arbitrary fixed ordering of the $(k-1)$-subsets of $\left\{\alpha_{1}, \ldots, \alpha_{r}\right\}$. Define

$$
f(\alpha, \varepsilon):=\mid\left\{i \in[n] \mid i \notin \alpha \text { and } \mu_{H}\left(\{i\} \cup e_{j}\right)=\varepsilon\left(e_{j}\right), \quad 1 \leq j \leq\left(\begin{array}{c}
r \\
k-1
\end{array}\right)\right\} \mid \text {. }
$$


Note that $N_{r+1}$ is the sum of exactly $N_{r}$ values $f_{r}(\alpha, \varepsilon)$. Namely, for each embedding $\lambda: G(r) \rightarrow H$, say with $\lambda\left(v_{j}\right)=\alpha_{j}, 1 \leq j \leq r, f(\alpha, \varepsilon)$ counts the number of ways of choosing $i \in[n]$ so that if we extend $\lambda$ to $V_{r+1}$ by setting $\lambda\left(v_{r+1}\right)=i$, and we define $\varepsilon\left(e_{j}\right)=\mu_{H}\left(\{i\} \cup e_{j}\right), 1 \leq j \leq\left(\begin{array}{c}r \\ k-1\end{array}\right)$, then $\lambda$ becomes an embedding of $G(r+1)$ into $H$. Also, there are just $2\left(\begin{array}{c}r \\ k-1\end{array}\right) n^{\underline{r}}$ quantities $f(\alpha, \varepsilon)$, since there are $n^{r}$ choices for $\alpha$ and $2^{\left(\begin{array}{c}r \\ k-1\end{array}\right)}$ choices for $\varepsilon$.

The next step is to compute the first and second moments of $f$. First, we have

$$
\bar{f}:=\frac{1}{2\left(\begin{array}{c}
r \\
k-1
\end{array}\right)_{n^{\underline{r}}}} \sum_{\alpha, \varepsilon} f(\alpha, \varepsilon)=(n-r) 2^{-\left(\begin{array}{c}
r \\
k-1
\end{array}\right) .} .
$$

In particular,

$$
\sum_{\alpha, \varepsilon} f(\alpha, \varepsilon)=n^{r+1}
$$

Next, define

$$
S_{r}:=\sum_{\alpha, \varepsilon} f^{2}(\alpha, \varepsilon)
$$

Claim 1.

$$
S_{r}=\sum_{x, y \in[m]} \#\left\{K_{r}^{(k-1)}<H_{x, y}^{-}\right\} ;
$$

$H_{x, y}^{-}$denotes the $(k-1)$-graph formed by restricting $H_{x, y}:=\overline{H(x) \nabla H(y)}$ to the vertex set $V^{-}:=V \backslash\{x, y\}$, and $K_{r}^{(m)}$ denotes the complete $m$-graph having $r$ vertices and all possible $\left(\begin{array}{c}m \\ r\end{array}\right) r$-sets as edges.

To see this, interpret $S_{r}$ in (6.5) as counting the number of ways of choosing $\alpha=\left(\alpha_{1}, \ldots, \alpha_{r}\right), \varepsilon=\left(\varepsilon\left(e_{1}\right), \ldots, \varepsilon\left(e_{z}\right)\right)$, and two other (ordered) vertices $x$ and $y$ in $[n] \backslash \alpha$ so that

$$
\mu_{H}\left(\{x\} \cup e_{j}\right)=\varepsilon\left(e_{j}\right)=\mu_{H}\left(\{y\} \cup e_{j}\right), \quad 1 \leq j \leq\left(\begin{array}{c}
r \\
k-1
\end{array}\right) .
$$

Summing over all $\varepsilon$ reduces this to requiring just that

$$
\mu_{H}\left(\{x\} \cup e_{j}\right)=\mu_{H}\left(\{y\} \cup e_{j}\right), \quad 1 \leq j \leq\left(\begin{array}{c}
r \\
k-1
\end{array}\right) .
$$

On the other hand, if we think of choosing $x$ and $y$ first, then by (6.7) $\left\{\alpha_{1}, \ldots, \alpha_{r}\right\}$ must span a $K_{r}^{(k-1)}$ in $H_{x, y}^{-}$. This proves Claim 1.

Of course,

$$
\#\left\{K_{r}^{(k-1)}<H_{x, y}^{-}\right\} \leq \#\left\{K_{r}^{(k-1)}<H_{x, y}\right\} .
$$

Claim 2. For $k \geq 2$,

$$
S_{r} \leq 2^{-\left(\begin{array}{c}
r \\
k-1
\end{array}\right)} n^{2} n^{r}+5 n^{r+2}(\operatorname{dev} H)^{2^{-(k-1)}} .
$$


Proof. First, assume $k=2$. Define

$$
\varepsilon(x, y):=\frac{1}{n} \sum_{z \in[n]} \mu_{H}(x, z) \mu_{H}(y, z) .
$$

Thus, $|\varepsilon(x, y)| \leq 1$ and

$$
\begin{aligned}
\operatorname{dev} H & =\frac{1}{n^{2}} \sum_{x, y} \varepsilon(x, y)^{2} \\
& \geq \frac{1}{n^{4}}\left(\sum_{x, y}|\varepsilon(x, y)|\right)^{2} \text { by Cauchy-Schwarz, }
\end{aligned}
$$

which implies

$$
\sum_{x, y}|\varepsilon(x, y)| \leq n^{2}(\operatorname{dev} H)^{1 / 2}
$$

Next, define

$$
s(x, y):=\frac{n}{2}(1+\varepsilon(x, y)) .
$$

Thus,

$$
\begin{aligned}
S_{r} & =\sum_{\alpha, \varepsilon} f^{2}(\alpha, \varepsilon)=\sum_{x, y} s(x, y)_{(r)} \\
& \leq \sum_{x, y} s(x, y)^{r}=\left(\frac{n}{2}\right)^{r} \sum_{x, y}(1+\varepsilon(x, y))^{r} \\
& =\left(\frac{n}{2}\right)^{r}\left\{n^{2}+\sum_{x, y} \sum_{j \geq 1}\left(\begin{array}{l}
r \\
j
\end{array}\right) \varepsilon(x, y)^{j}\right\} \\
& \leq\left(\frac{n}{2}\right)^{r}\left\{n^{2}+2^{r} \sum_{x, y}|\varepsilon(x, y)|\right\} \\
& \leq \frac{n^{r+2}}{2^{r}}+n^{r+2}(\operatorname{dev} H)^{1 / 2} \text { by }(6.10) \\
& \leq \frac{n^{2} n^{r}}{2^{r}}+5 n^{r+2}(\operatorname{dev} H)^{1 / 2}
\end{aligned}
$$

as required by (6.9) for $k=2$, where we have used (4.3) and the fact that

$$
\frac{n^{r}}{n^{r}} \geq 1-\frac{1}{n}\left(\begin{array}{l}
r \\
2
\end{array}\right) \text {. }
$$

We now observe that the remainder of the proof of Theorem 6.1 for the case $k=2$ can be completed as written (following the end of Claim 2).

In general, for some $k>2$, the proof of Claim 2 for $k$ will require the use of Theorem 6.1 for $k-1$. So we can assume for $k>2$ that Theorem 6.1 holds for $k-1$, and we will complete the proof of (6.9) for this value of $k$. 
By (6.1) for $k-1$, we have

$$
\begin{aligned}
S_{r} & \leq \sum_{x, y} \#\left\{K_{r}^{(k-1)}<H_{x, y}\right\} \\
& \leq 2^{-\left(\begin{array}{c}
r \\
k-1
\end{array}\right)} n^{2} r^{\underline{r}}+5 n^{r+2}\left(\frac{1}{n^{2}} \sum_{x, y} \operatorname{dev} H_{x, y}\right)^{2^{-(k-1)}} \\
& \quad \text { by repeated application of Cauchy-Schwarz } \\
& =2^{-\left(\begin{array}{c}
r \\
k-1
\end{array}\right)} n^{2} n^{\underline{r}}+5 n^{r+2}(\operatorname{dev} H)^{2^{-k-1}}
\end{aligned}
$$

by Fact 4.0 and Fact 4.3. This completes the proof of Claim 6.2.

We now compute the variance of $f$.

$$
\begin{aligned}
& \operatorname{Var} f=\sum_{\alpha, \varepsilon}(f(\alpha, \varepsilon)-\bar{f})^{2}=\sum_{\alpha, \varepsilon} f^{2}(\alpha, \varepsilon)-\sum_{\alpha, \varepsilon} \bar{f}^{2} \\
& =S_{r}-\frac{(n-r)^{2}}{2^{2\left(\begin{array}{c}
r \\
k-1
\end{array}\right)}} 2^{-\left(\begin{array}{c}
r \\
k-1
\end{array}\right)} n^{r}=S_{r}-\frac{(n-2)^{2} n^{r}}{2^{\left(\begin{array}{c}
r \\
k-1
\end{array}\right)}} \\
& \leq \frac{n^{2} n^{\underline{r}}}{2{ }^{\left(\begin{array}{c}
r \\
k-1
\end{array}\right)}}+5 n^{r+2}(\operatorname{dev} H)^{2^{-(k-1)}}-\frac{(n-r)^{2} n^{r}}{2^{\left(\begin{array}{c}
r \\
k-1
\end{array}\right)}} \\
& =\frac{r(2 n-r) n^{\underline{r}}}{2^{\left(\begin{array}{c}
r \\
k-1
\end{array}\right)}}+5 n^{r+2}(\operatorname{dev} H)^{2^{-(k-1)}} \text {. }
\end{aligned}
$$

Also, since

$$
N_{r+1}=\sum_{\substack{N_{r} \text { choices } \\ \text { of }(\alpha, \varepsilon)}} f(\alpha, \varepsilon)
$$

then

$$
\begin{aligned}
\left|N_{r+1}-N_{r} \bar{f}\right|^{2} & =\left|\sum_{N_{r} \text { terms }}(f(\alpha, \varepsilon)-\bar{f})\right|^{2} \\
& \leq N_{r} \sum_{N_{r} \text { terms }}(f(\alpha, \varepsilon)-\bar{f})^{2} \quad \text { by Cauchy-Schwarz } \\
& \leq N_{r} \sum_{\text {all }(\alpha, \varepsilon)}(f(\alpha, \varepsilon)-\bar{f})^{2}=N_{r} \operatorname{Var} f \\
& \leq N_{r}\left\{5 n^{r+2}(\operatorname{dev} H)^{2^{-(k-1)}}+\frac{r(2 n-r) n^{\underline{r}}}{\left.2^{\left(\begin{array}{c}
r \\
k-1
\end{array}\right)}\right\}} .\right.
\end{aligned}
$$

Thus,

$$
\left|N_{r+1}-N_{r} \bar{f}\right| \leq N_{r}^{1 / 2}\left\{5 n^{r+2}(\operatorname{dev} H)^{2^{-(k-1)}}+\frac{r(2 n-r) n^{\underline{r}}}{2^{\left(\begin{array}{c}
r \\
k-1
\end{array}\right)}}\right\}^{1 / 2}
$$


Now, by induction on $r$

$$
\left|N_{r}-\frac{n^{\underline{r}}}{2\left(\begin{array}{l}
r \\
k
\end{array}\right)}\right| \leq 5 n^{r}(\operatorname{dev} H)^{2^{-k}} .
$$

Since $\bar{f}=(n-r) / 2^{\left(\begin{array}{c}r \\ k-1\end{array}\right)}$ by (6.3) we obtain

$$
\begin{aligned}
\mid N_{r+1}-\frac{n^{r+1}}{2^{\left(\begin{array}{c}
r+1 \\
k
\end{array}\right)} \mid \leq} & N_{r}^{1 / 2}\left\{5 n^{r+2}(\operatorname{dev} H)^{2^{-(k-1)}}+\frac{r(2 n-r) n^{\underline{r}}}{\left.2^{\left(\begin{array}{c}
r \\
k-1
\end{array}\right)}\right\}^{1 / 2}}\right. \\
& +\frac{5(n-r) n^{r}(\operatorname{dev} H)^{2^{-k}}}{2^{\left(\begin{array}{c}
r \\
k-1
\end{array}\right)}} \\
\leq & 5 n^{r+1}(\operatorname{dev} H)^{2^{-k}}
\end{aligned}
$$

where the final inequality follows by straightforward computation using the assumptions $1 \leq k-1 \leq r \leq \sqrt{n}$ and the (trivial) estimate $N_{r} \leq n^{r}$.

This therefore completes the induction step and (6.1) follows. Thus, Theorem 6.1 is proved.

We should point out that the basic structure of this proof has its roots in the seminal paper of Wilson [Wi72].

Theorem 6.1 has a quite unexpected consequence. What it asserts in essence is that the smaller $\operatorname{dev} H$ is, the closer $\#\{G(t)<H\}$ is (for any fixed $G(t)$ ) to what is expected, namely $2^{-\left(\begin{array}{l}t \\ k\end{array}\right)} n^{\underline{t}}$. However, $\operatorname{dev} H$ only depends on $\#\{\mathrm{EPO}<H\}$ and $\#\{\mathrm{OPO}<H\}$, which in turn, depend on the quantities $\#\{G(2 k)<H\}$, as $G(2 k)$ ranges over all $2 k$-vertex $k$-graphs. Thus, if each of the $2 k$-vertex $k$-graphs occur as induced subgraphs of $H$ about the "correct" number of times, then in fact so do all the $t$-vertex $k$-graphs, as well! Of course, for a fixed $H$, the larger $t$ becomes, the larger the variation of the actual count from the expected value becomes. (Theorem 6.1 gives a quantitative statement of this phenomenon.) As will be seen in $\S 7$, the value $2 k$ is in fact sharp for $k$-graphs. That is, for each $k$ there is a family of $k$-graphs $H(n), n \rightarrow \infty$, so that:

(i) For all $G(2 k-1)$,

$$
\#\{G(2 k-1)<H(n)\}=(1+o(1)) \frac{n^{\frac{2 k-1}{2 k-1}}}{2^{\left(\begin{array}{c}
2 k \\
k
\end{array}\right)}} ;
$$

(ii) for some $G(2 k)$ and some $\varepsilon>0$,

$$
\#\{G(2 k)<H(n)\}<(1-\varepsilon) \frac{n^{\underline{2 k}}}{2^{\left(\begin{array}{c}
2 k \\
k
\end{array}\right)}}, \quad n>n_{0}(\varepsilon) .
$$

In fact, for $k \neq 2^{s}$, we construct in $\S 7$ examples of $k$-graphs $H(n)$ that satisfy 
(i) and

$\left(\mathrm{ii}^{\prime}\right)$ for some $G(2 k)$,

$$
\#\{G(2 k)<H(n)\}=0 .
$$

Such $k$-graphs $H(n)$ clearly deviate from behaving like random $k$-graphs in a very striking way. It follows from (6.1) that if

$$
\frac{1}{n}<\left(1-5 \cdot 2^{\left(\begin{array}{l}
t \\
k
\end{array}\right)}(\operatorname{dev} H)^{2^{-k}}\right)\left(\begin{array}{l}
t \\
2
\end{array}\right)^{-1}
$$

then any $G(t)$ is an induced subgraph of $H=H(n)$.

Suppose $\operatorname{dev} H<1$ (the very special $k$-graphs having deviation 1 are characterized in $\S 11)$. By (4.8) with $l=k$ and $H_{i}=H, 1 \leq i \leq m$, we have for $H^{\square m}:=\square_{i=1}^{m} H$,

$$
\operatorname{dev} H^{\square m}=(\operatorname{dev} H)^{m}, \quad\left|H^{\square m}\right|=|H|^{m}=n^{m} .
$$

It then follows from (6.13) that for $m$ large enough, e.g., $m>\left(2 n^{2} t\right)^{k}$, we have $\#\left\{G(t)<H^{\square m}\right\}>0$. However, we can draw the same conclusion for a smaller value of $m$ by the following direct construction.

Proposition 6.2. If $\operatorname{dev} H<1$ then $\#\left\{G(t)<H^{\square m}\right\}>0$ provided that $m \geq\left(\begin{array}{c}t-1 \\ k-1\end{array}\right) 2^{k-1}$.

Proof. By hypothesis, $H$ must contain an OPO, i.e., $2 k$ vertices $x_{i}<y_{i}$, $1 \leq i \leq k$, so that

$$
\begin{aligned}
& \prod_{\substack{z_{i} \in\left\{x_{i}, y_{i}\right\} \\
1 \leq i \leq k-1}} \bar{\mu}_{H}\left(z_{1}, \ldots, z_{k-1}, x_{k}\right)=-1, \\
& \prod_{\substack{z_{i} \in\left\{x_{i}, y_{i}\right\} \\
1 \leq i \leq k-1}} \bar{\mu}_{H}\left(z_{1}, \ldots, z_{k-1}, y_{k}\right)=1 .
\end{aligned}
$$

Let $m_{0}:=\left(\begin{array}{c}t-1 \\ k-1\end{array}\right) 2^{k-1}$ and let $W=\left\{w_{1}<w_{2}<\cdots<w_{t}\right\}$ denote the vertex set of $G(t)$. Also, let $W^{\prime}$ denote $W \backslash\left\{w_{t}\right\}$. The plan will be to map each $w \in W$ to an $m_{0}$-tuple $\bar{w}$ as follows. Let $\left(U_{1}, U_{2}, \ldots, U_{\left(\begin{array}{c}t-1 \\ j-1\end{array}\right)}\right)$ be some fixed ordering of $\left(\begin{array}{c}W^{\prime} \\ k-1\end{array}\right)$. For $U_{j}$, we reserve the $2^{k-1}$ coordinate positions $\left\{(j-1) 2^{k-1}+i \mid 0 \leq i<2^{k-1}\right\}$. Write $U_{j}=\left\{u_{1}<\cdots<u_{k-1}\right\}$. Let $\bar{w}(j, i)$, $0 \leq i<2^{k-1}$, be the values $\bar{w}$ is assigned in these coordinates. Then $\bar{w}(j, i)$ is defined as follows.

(i) $w>u_{k-1}$. Then

$$
\bar{w}(j, i)= \begin{cases}x_{k} & \text { if } U_{j} \cup\{w\} \text { is an edge of } G(t), \\ y_{k} & \text { if } U_{j} \cup\{w\} \text { is not an edge of } G(t) .\end{cases}
$$

(ii) $w<u_{k-1}, w \notin U_{j}$. Then

$$
\bar{w}(j, i)=y_{k} .
$$


(iii) $w=u_{r} \in U_{j}$. Then

$\bar{w}(j, i)= \begin{cases}x_{k} & \text { if the } r \text { th digit of the binary expansion of } i \text { is } 1, \\ y_{k} & \text { if the } r \text { th digit of the binary expansion of } i \text { is } 0 .\end{cases}$

With this assignment for each $U_{j} \in\left(\begin{array}{c}W^{\prime} \\ k-1\end{array}\right)$, we have defined a mapping $\lambda: W \rightarrow$ $\left\{x_{1}, y_{1}, \ldots, x_{k}, y_{k}\right\}^{m_{0}}$. It is now simply a matter of checking to see that $\lambda$ induces a copy of $G(t)$ in $H^{\square m_{0}}$, and the claim is proved.

A small example may help to clarify this construction.

Example. $k=3, t=5, m_{0}=24, W=\{1,2,3,4,5\}$, and $G(5)$ has edges $\{1,2,3\},\{1,2,5\},\{1,3,4\},\{2,4,5\}$.

\begin{tabular}{c|cccccccccccc} 
& \multicolumn{1}{|c}{12} & \multicolumn{1}{c}{13} & \multicolumn{1}{c}{14} \\
\hline$w$ & 0 & 1 & 2 & 3 & 4 & 5 & 6 & 7 & 8 & 9 & 10 & 11 \\
\hline 1 & $y_{1}$ & $y_{1}$ & $x_{1}$ & $x_{1}$ & $y_{1}$ & $y_{1}$ & $x_{1}$ & $x_{1}$ & $y_{1}$ & $y_{1}$ & $x_{1}$ & $x_{1}$ \\
2 & $y_{2}$ & $x_{2}$ & $y_{2}$ & $x_{2}$ & $y_{3}$ & $y_{3}$ & $y_{3}$ & $y_{3}$ & $y_{3}$ & $y_{3}$ & $y_{3}$ & $y_{3}$ \\
3 & $x_{3}$ & $x_{3}$ & $x_{3}$ & $x_{3}$ & $y_{2}$ & $x_{2}$ & $y_{2}$ & $x_{2}$ & $y_{3}$ & $y_{3}$ & $y_{3}$ & $y_{3}$ \\
4 & $y_{3}$ & $y_{3}$ & $y_{3}$ & $y_{3}$ & $x_{3}$ & $x_{3}$ & $x_{3}$ & $x_{3}$ & $y_{2}$ & $x_{2}$ & $y_{2}$ & $x_{2}$ \\
5 & $x_{3}$ & $x_{3}$ & $x_{3}$ & $x_{3}$ & $y_{3}$ & $y_{3}$ & $y_{3}$ & $y_{3}$ & $y_{3}$ & $y_{3}$ & $y_{3}$ & $y_{3}$
\end{tabular}

\begin{tabular}{c|cccccccccccc} 
& \multicolumn{9}{|c}{23} & \multicolumn{1}{c}{24} & \multicolumn{1}{c}{34} \\
\hline$w$ & 12 & 13 & 14 & 15 & 16 & 17 & 18 & 19 & 20 & 21 & 22 & 23 \\
\hline 1 & $y_{3}$ & $y_{3}$ & $y_{3}$ & $y_{3}$ & $y_{3}$ & $y_{3}$ & $y_{3}$ & $y_{3}$ & $y_{3}$ & $y_{3}$ & $y_{3}$ & $y_{3}$ \\
2 & $y_{1}$ & $y_{1}$ & $x_{1}$ & $x_{1}$ & $y_{1}$ & $y_{1}$ & $x_{1}$ & $x_{1}$ & $y_{3}$ & $y_{3}$ & $y_{3}$ & $y_{3}$ \\
3 & $y_{2}$ & $x_{2}$ & $y_{2}$ & $x_{2}$ & $y_{3}$ & $y_{3}$ & $y_{3}$ & $y_{3}$ & $y_{1}$ & $y_{1}$ & $x_{1}$ & $x_{1}$ \\
4 & $y_{3}$ & $y_{3}$ & $y_{3}$ & $y_{3}$ & $y_{2}$ & $x_{2}$ & $y_{2}$ & $x_{2}$ & $y_{2}$ & $x_{2}$ & $y_{2}$ & $x_{2}$ \\
5 & $y_{3}$ & $y_{3}$ & $y_{3}$ & $y_{3}$ & $x_{3}$ & $x_{3}$ & $x_{3}$ & $x_{3}$ & $y_{3}$ & $y_{3}$ & $y_{3}$ & $y_{3}$
\end{tabular}

\section{7. $k$-GRAPHS WITH THE CORRECT DENSITY OF SMALL SUBGRAPHS}

We saw in the preceding section that if a $k$-graph $H(n)$ contains all possible $2 k$-vertex $k$-graphs $G(2 k)$ as induced subgraphs asymptotically equally often,. then in fact this must also hold for all $t$-vertex $k$-graphs $G(t)$, for any fixed $t$, as well. In this section we show that the value $2 k$ is critical for such a conclusion to hold. Specifically, we will prove

Theorem 7.1. Fix $2 \leq k \leq l \leq 2 k-1$. Then there exists a family of $k$-graphs $H(n)$ such that

(i) for any $G(l), \#\{G(l)<H(n)\}=(1+o(1)) n^{l} / 2\left(\begin{array}{l}l \\ k\end{array}\right)$;

(ii) for some $G(l+1), \#\{G(l+1)<H(n)\} \neq(1+o(1)) n^{l+1} / 2\left(\begin{array}{c}l+1 \\ k\end{array}\right)$.

Proof. We will first deal with the case $l=2 k-1$. The general case will then follow in the same way. Fix some vertex set $V$ of size $n$. For $1 \leq j \leq k-1$, let 
$H_{1 / 2}^{(j)}$ be a random $j$-graph $\left(V, \chi_{j}\right)$, where in this section we use the additive edge function $\chi_{j}$. Define $H_{j}$ to be the $k$-graph $\left(V, \chi_{j}^{\prime}\right)$ given by:

$$
\text { for } X \in\left(\begin{array}{l}
V \\
k
\end{array}\right), \quad \chi_{j}^{\prime}(X):=\sum_{Y \in\left(\begin{array}{c}
X \\
j
\end{array}\right)} \chi_{j}(Y)(\bmod 2) \text {. }
$$

That is, $X$ is an edge of $H_{j}$ if and only if $X$ contains an odd number of edges $Y$ of $H_{1 / 2}^{(j)}$. Form the symmetric difference $k$-graph

$$
H^{*}=H^{*}(n)=\nabla_{j=1}^{k-1} H_{j}=\left(V, \chi^{*}\right) .
$$

We claim that $H^{*}(n)$ satisfies the desired conclusions, except when $k=2^{t}$, in which case an additional step is required.

So we first assume $k \neq 2^{t}$. Consider an arbitrary fixed set $W=\left\{w_{1}, \ldots\right.$, $\left.w_{2 k-1}\right\}$ of $2 k-1$ vertices in $V$. Form the matrix $M$ with rows indexed by $X \in\left(\begin{array}{c}W \\ k\end{array}\right)$ and columns indexed by $Y_{j}=\left(\begin{array}{c}W \\ j\end{array}\right), 1 \leq j \leq k-1$. Thus $M$ has size $\left(\begin{array}{c}2 k-1 \\ k\end{array}\right)$ by $2^{k-1}-1$. The $\left(X, Y_{j}\right)$-entry $M\left(X, Y_{j}\right)$ of $M$ is defined to be 1 if $Y_{j} \subset X$ and 0 , otherwise. We can view each column $C\left(Y_{j}\right)$ as a function mapping $\left(\begin{array}{c}W \\ k\end{array}\right)$ to $\{0,1\}$ by defining

$$
C\left(Y_{j}\right)(X)=M\left(X, Y_{j}\right), \quad X \in\left(\begin{array}{c}
W \\
k
\end{array}\right) .
$$

Furthermore it is easy to see that

$$
\sum_{Y_{j} \in\left(\begin{array}{c}
W \\
j
\end{array}\right)} C\left(Y_{j}\right)=\chi_{j}^{\prime}
$$

so that

$$
\sum_{1 \leq j \leq k-1} \sum_{Y_{j} \in\left(\begin{array}{c}
W \\
j
\end{array}\right)} C\left(Y_{j}\right)=\chi^{*}
$$

The key fact we now apply is a result of Wilson [Wi90] that asserts that (for $\left.k \neq 2^{t}\right) \quad M$ has full rank mod 2, which in this case is $\left(\begin{array}{c}2 k-1 \\ k\end{array}\right)$. Actually, Wilson's result implies that if we adjoin the all 1 's column $\overline{1}$ to $M$, forming $M^{+}$, then $M^{+}$has mod 2 rank equal to $\left(\begin{array}{c}2 k-1 \\ k\end{array}\right)$. However, for $k \neq 2^{t}$ some $i$ with $1 \leq i \leq k-1$ has $\left(\begin{array}{c}k \\ i\end{array}\right)$ odd. Summing all the columns $C\left(Y_{i}\right), Y_{i} \in\left(\begin{array}{c}V \\ i\end{array}\right)$, yields $\overline{1}$ so that in fact $\operatorname{rank}_{2} M=\operatorname{rank}_{2} M^{+}=\left(\begin{array}{c}2 k-1 \\ k\end{array}\right)$. As $W$ ranges over all $(2 k-1)$ element subsets of $V$, since the edges of the various corresponding $H_{1 / 2}^{(j)}$ are chosen independently (and uniformly), then an easy argument shows that each of the possible $\left(\begin{array}{c}2 k-1 \\ k\end{array}\right)(0,1)$-vectors occurs $(1+o(1)) n^{2 k-1} / 2^{\left(\begin{array}{c}2 k-1 \\ k\end{array}\right)}$ times as $n \rightarrow \infty$. But this just means that for almost all choices of the $H_{1 / 2}^{(j)}$ each of the possible $k$-graphs $G(2 k-1)$ on $2 k-1$ vertices occurs $(1+o(1)) n^{2 k-1} / 2\left(\begin{array}{c}2 k-1 \\ k\end{array}\right)$ times as an induced subgraph of $H^{*}(n)$, as claimed. 
In the case $k=2^{t}$ for some $t$, we form the $k$-graph $H^{+}$by taking the two disjoint $k$-graphs $H^{*}$ and its complement $\overline{H^{*}}$, and placing a random $k$-graph between them. That is, if $H^{*}=\left(V, \chi^{*}\right), \bar{H}^{*}=\left(V^{\prime}, \bar{\chi}^{*}\right), V \cap V^{\prime}=\varnothing$, then $H^{+}=H^{+}(2 n)=\left(V \cup V^{\prime}, \chi^{+}\right)$with

$$
\chi^{+}(X)= \begin{cases}\chi^{*}(X) & \text { for } X \subseteq V \\ \bar{\chi}^{*}(X) & \text { for } X \subseteq V^{\prime} \\ 0 \text { or } 1 & \text { with probability } 1 / 2 \text { otherwise }\end{cases}
$$

for $X \in\left(\begin{array}{c}V \cup V^{\prime} \\ k\end{array}\right)$. An easy argument shows that

$$
\#\left\{G(2 k-1)<H^{+}(2 n)\right\}=(1+o(1))(2 n)^{2 k-1} 2^{-\left(\begin{array}{c}
2 k-1 \\
k
\end{array}\right)}
$$

for almost all choices defining $H^{+}$.

To see that there are $2 k$-vertex $k$-graphs $G(2 k)$ satisfying (ii) (for $l=$ $2 k-1)$, it is enough to show that $\operatorname{dev} H^{*}$ and $\operatorname{dev} H^{+}$are bounded away from 0 (independent of $n$ ). Indeed we show in $\S 11$ that $\operatorname{dev} H^{*}=1$ and, consequently, for almost all choices of $H^{+}, \operatorname{dev} H^{+} \geq(1+o(1)) 2^{1-2 k}$.

Now, we treat the case of general $l$. Of course, for $l=k$ the conclusion is immediate so we may assume $l>k$. We apply the same constructions as in the preceding case $l=2 k-1$, but now with rows of $M$ indexed by sets $X \in\left(\begin{array}{l}V \\ k\end{array}\right)$, and columns indexed by sets $Y_{j} \in V_{j}, 1 \leq j \leq l-k$ (where $|V|=l$ ). As before, Wilson's result applies to the augmented matrix $M^{+}$(with the all 1's column adjoined), with the conclusion that $M^{+}$has mod 2 rank equal to $\left(\begin{array}{l}l \\ k\end{array}\right)$. Thus, it follows that when $k \neq 2^{t}$ then $M$ itself has mod 2 rank equal to $\left(\begin{array}{l}l \\ k\end{array}\right)$ and the analogous construction of $H^{*}$ gives us the desired $k$-graph, while for $k=2^{t}$ the "doubling" construction of $H^{+}$(using $H^{*}$ and $\overline{H^{*}}$ ) works here as well. It follows as before that $\operatorname{dev} H^{*}=1$, and $\operatorname{dev} H^{+}$almost always exceeds $(1+o(1)) 2^{1-k-l}$. This completes the proof.

\section{DisCREPANCY}

In this section we relate deviation to another measure of randomness for $k$ graphs called discrepancy. This is a natural generalization of the well-studied concept of the discrepancy of a graph [ES72, ES74] and was suggested (for $k=3$ ) by Frankl and Rödl [FR89] as a possible quasi-random property. We will show here that deviation and discrepancy are indeed intimately related, and in fact, one can be small only if the other one is.

To begin with, we need to define discrepancy. Let $H$ be an arbitrary $k$-graph on an $n$-vertex set $V$ and with edge set $E(H)$. For a $(k-1)$-graph $G$ on $V$ with edge set $E(G)$, we define

$$
\begin{aligned}
& E(H, G):=\left\{X \in E(H) \mid\left(\begin{array}{c}
X \\
k-1
\end{array}\right) \subseteq E(G)\right\}, \\
& e(H, G):=k !|E(H, G)| .
\end{aligned}
$$


Thus, $e(H, G)$ counts the number of ordered subsets in $E(H, G)$. Finally, we define the discrepancy of $H$, denoted by $\operatorname{disc} H$, by

$$
\operatorname{disc} H:=\frac{1}{n^{k}} \max _{G}|e(H, G)-e(\bar{H}, G)|,
$$

where the max is taken over all $(k-1)$-graphs $G$ on $V$.

For $k=1$, we take by convention

$$
\operatorname{disc} H=\frac{1}{n}|e(H)-e(\bar{H})|,
$$

where, as usual, $e(K)$ denotes the number of edges of $K$.

For $k=2$, it follows from (8.2) that

$$
\operatorname{disc} H=\frac{2}{n^{2}} \max _{W \subset V}|e(H[W])-e(\bar{H}[W])| .
$$

Thus, the discrepancy of a 2-graph just measures the maximum imbalance between edges and non-edges over all its induced subgraphs.

It is easily proved that almost all random $k$-graphs $H_{1 / 2}(n)$ satisfy

$$
\operatorname{disc} H_{1 / 2}(n)=o(1), \quad n \rightarrow \infty,
$$

and we have seen by Fact 5.1 that almost all $H_{1 / 2}(n)$ satisfy

$$
\operatorname{dev} H_{1 / 2}(n)=o(1), \quad n \rightarrow \infty .
$$

What was actually quite unexpected was that for arbitrary $k$-graphs $H,(8.5)$ and (8.6) are equivalent.

The following result states this in a more quantitative form.

Theorem 8.1. For any $k$-graph $H=\left(V, \mu_{H}\right)$ :

(i) $\operatorname{disc} H \leq(\operatorname{dev} H)^{2^{-k}}$;

(ii) $\operatorname{dev} H \leq 4^{k}(\operatorname{disc} H)^{2^{-k}}$.

Proof. First observe that for $k=1$ the desired conclusions are immediate since in this case

$$
\begin{aligned}
\operatorname{dev} H & =\frac{1}{n^{2}} \sum_{x, y} \mu_{H}(x) \mu_{H}(y) \\
& =\left(\frac{1}{n} \sum_{x} \mu_{H}(x)\right)^{2}=(\operatorname{disc} H)^{2} .
\end{aligned}
$$

Hence we assume $k \geq 2$.

We first prove (i). Let $G$ be an arbitrary $(k-1)$-graph on $V$ with edge set 
$E(G)$. By definition,

$$
\begin{aligned}
n^{2 k} \operatorname{dev} H & =\sum_{\substack{u_{i}(0), u_{i}(1) \\
1 \leq i \leq k}} \prod_{\substack{\varepsilon_{j} \in\{0,1\} \\
1 \leq j \leq k}} \bar{\mu}_{H}\left(u_{1}\left(\varepsilon_{1}\right), \ldots, u_{k}\left(\varepsilon_{k}\right)\right) \\
& =\sum_{\substack{u_{i}(0), u_{i}(1) \\
i \neq 1}}\left(\sum_{\substack { v \in V \\
\begin{subarray}{c}{\varepsilon_{j} \\
j \neq 1{ v \in V \\
\begin{subarray} { c } { \varepsilon _ { j } \\
j \neq 1 } }\end{subarray}} \prod_{H}\left(v, u_{2}\left(\varepsilon_{2}\right), \ldots, u_{k}\left(\varepsilon_{k}\right)\right)\right)^{2} \\
& \geq \sum_{\substack{u_{i}(0), u_{i}(1) \\
i \neq 1}}^{(1)}\left(\sum_{v} \prod_{\substack{\varepsilon_{j} \\
j \neq 1}} \bar{\mu}_{H}\left(v, u_{2}\left(\varepsilon_{2}\right), \ldots, u_{k}\left(\varepsilon_{k}\right)\right)\right)^{2}
\end{aligned}
$$

where $\sum^{(1)}$ denotes a sum over all choices of $u_{i}(0), u_{i}(1), i \neq 1$, such that $\left\{u_{2}\left(\varepsilon_{2}\right), \ldots, u_{k}\left(\varepsilon_{k}\right)\right\}$ is an edge of $G$ for all choices of $\varepsilon_{j} \in\{0,1\}, 2 \leq j \leq k$,

$$
\begin{aligned}
& =\sum_{u_{i}(0), u_{i}(1)}^{(1)} \prod_{\varepsilon_{j}} \bar{\mu}_{H}\left(u_{1}\left(\varepsilon_{1}\right), \ldots, u_{k}\left(\varepsilon_{k}\right)\right) \\
& =\sum_{\substack{u_{i}(0), u_{i}(1) \\
i \neq 2}}\left(\sum_{v}^{(1)} \prod_{\substack{\varepsilon_{j} \\
j \neq 2}} \bar{\mu}_{H}\left(u_{1}\left(\varepsilon_{1}\right), v, u_{3}\left(\varepsilon_{3}\right), \ldots, u_{k}\left(\varepsilon_{k}\right)\right)\right)^{2} \\
& \geq \sum_{\substack{u_{i}(0), u_{i}(1) \\
i \neq 2}}^{(2)}\left(\sum_{v}^{(1)} \prod_{\substack{\varepsilon_{j} \\
j \neq 2}} \bar{\mu}_{H}\left(u_{1}\left(\varepsilon_{1}\right), v, \ldots, u_{k}\left(\varepsilon_{k}\right)\right)\right)^{2}
\end{aligned}
$$

where $\sum^{(2)}$ denotes a sum over all choices of $u_{i}(0), u_{i}(1), i \neq 2$, such that all $\left\{u_{1}\left(\varepsilon_{1}\right), u_{3}\left(\varepsilon_{3}\right), \ldots, u_{k}\left(\varepsilon_{k}\right)\right\} \in E(G)$ for all $\varepsilon_{j} \in\{0,1\}$

$$
=\sum_{u_{i}(0), u_{i}(1)}^{(1,2)} \prod_{\varepsilon_{j}} \bar{\mu}_{H}\left(u_{1}\left(\varepsilon_{1}\right), \ldots, u_{k}\left(\varepsilon_{k}\right)\right)
$$

where $\sum^{(1,2)}$ denotes a sum over all choices of $u_{i}(0), u_{i}(1)$ such that all $\left\{u_{2}\left(\varepsilon_{2}\right)\right.$, $\left.u_{3}\left(\varepsilon_{3}\right), \ldots, u_{k}\left(\varepsilon_{k}\right)\right\}$ and all $\left\{u_{1}\left(\varepsilon_{1}\right), u_{3}\left(\varepsilon_{3}\right), \ldots, u_{k}\left(\varepsilon_{k}\right)\right\}$ are edges of $G$ for all . $\varepsilon_{j} \in\{0,1\}$

$$
\begin{gathered}
\geq \sum_{u_{i}(0), u_{i}(1)}^{(1,2,3)} \prod_{\varepsilon_{j}} \bar{\mu}_{H}\left(u_{1}\left(\varepsilon_{1}\right), \ldots, u_{k}\left(\varepsilon_{k}\right)\right) \\
\vdots \\
\geq \sum_{u_{i}(0), u_{i}(1)}^{(1,2, \ldots, k)} \prod_{\varepsilon_{j}} \bar{\mu}_{H}\left(u_{1}\left(\varepsilon_{1}\right), \ldots, u_{k}\left(\varepsilon_{k}\right)\right)
\end{gathered}
$$

where the final sum is over all choices of $u_{i}(0), u_{i}(1)$ such that every $(k-1)$ subset of all $\left\{u_{1}\left(\varepsilon_{1}\right), \ldots, u_{k}\left(\varepsilon_{k}\right)\right\}, \varepsilon_{j} \in\{0,1\}$, are edges of $G$. We now repeatedly apply the Cauchy-Schwarz inequality. 


$$
\begin{aligned}
& \sum_{u_{i}(0), u_{i}(1)}^{(1,2, \ldots, k)} \prod_{\varepsilon_{j}} \bar{\mu}_{H}\left(u_{1}\left(\varepsilon_{1}\right), \ldots, u_{k}\left(\varepsilon_{k}\right)\right) \\
& =\sum_{\substack{u_{i}(0), u_{i}(1) \\
i \neq 1}}\left(\sum_{v_{1}}^{(1,2, \ldots, k)} \prod_{\substack{\varepsilon_{j} \\
j \neq 1}} \bar{\mu}_{H}\left(v_{1}, u_{2}\left(\varepsilon_{2}\right), \ldots, u_{k}\left(\varepsilon_{k}\right)\right)\right)^{2} \\
& \geq \frac{1}{n^{2 k-2}}\left(\sum_{\substack{v_{1} \\
u_{i}(0), u_{i}(1) \\
i>1}}^{(1,2, \ldots, k)} \prod_{\substack{\varepsilon_{j} \\
j>1}} \bar{\mu}_{H}\left(v_{1}, u_{2}\left(\varepsilon_{2}\right), \ldots, u_{k}\left(\varepsilon_{k}\right)\right)\right)^{2} \\
& =\frac{1}{n^{2 k-2}}\left(\sum_{\substack{v_{1} \\
u_{i}(0), u_{i}(1) \\
i>2}}\left(\sum_{v_{2}}^{(1,2, \ldots, k)} \prod_{\substack{\varepsilon_{j} \\
j>2}} \bar{\mu}_{H}\left(v_{1}, v_{2}, u_{3}\left(\varepsilon_{3}\right), \ldots\right)\right)^{2}\right)^{2} \\
& =\frac{1}{n^{2 k-2}} \cdot \frac{1}{n^{2(2 k-3)}}\left(\sum_{\substack{v_{1}, v_{2} \\
u_{i}(0), u_{i}(1) \\
i>2}}^{(1,2, \ldots, k)} \prod_{\substack{\varepsilon_{j} \\
j>2}} \bar{\mu}_{H}\left(v_{1}, v_{2}, u_{3}\left(\varepsilon_{3}\right), \ldots\right)\right)^{4} \\
& \geq \frac{1}{n^{2 k-2}} \cdot \frac{1}{n^{2(2 k-3)}} \cdots \frac{1}{n^{2^{t-1}(2 k-t-1)}} \\
& \times\left(\sum_{\substack{v_{1}, \ldots, v_{t} \\
u_{i}(0), u_{i}(1) \\
i>t}}^{(1,2, \ldots, k)} \prod_{\substack{\varepsilon_{j} \\
j>t}} \bar{\mu}_{H}\left(v_{1}, \ldots, v_{t}, u_{t+1}\left(\varepsilon_{t+1}\right), \ldots\right)\right)^{2^{t}} \\
& \geq \frac{1}{n^{k\left(2^{k}-2\right)}}\left(\sum_{v_{1}, \ldots, v_{t}}^{(1,2, \ldots, k)} \bar{\mu}_{H}\left(v_{1}, \ldots, v_{k}\right)\right)^{2^{k}} \\
& =\frac{1}{n^{k\left(2^{k}-2\right)}}(e(H, G)-e(\bar{H}, G))^{2^{k}}
\end{aligned}
$$

since all $(k-1)$-subsets of each choice of $\left\{v_{1}, \ldots, v_{k}\right\}$ are edges of $G$.

Thus

$$
\begin{aligned}
|e(H, G)-e(\bar{H}, G)| & \leq\left(n^{k\left(2^{k}-2\right)} \cdot n^{2 k} \operatorname{dev} H\right)^{1 / 2^{k}} \\
& \leq n^{k}(\operatorname{dev} H)^{1 / 2^{k}}
\end{aligned}
$$

Since $G$ was arbitrary then this implies

$$
\operatorname{disc} H \leq(\operatorname{dev} H)^{1 / 2^{k}}
$$

as required.

We next prove (ii) by induction on $k$. We first remark that for $k=2$, (ii) follows directly from results in [CGW, Fact 9], so we will assume $k \geq 3$. 
Assume that for every $(k-1)$-graph $G$ on $V$, we have

$$
|e(H, G)-e(\bar{H}, G)|<2 \varepsilon^{2} n^{k} .
$$

We will show

$$
\operatorname{dev} H<4^{k} \varepsilon^{1 / 2^{k}}
$$

For each $u \in V$, define

$$
\begin{aligned}
S(u) & :=\left\{v \in V \mid \operatorname{dev} H_{u, v} \geq\left(4^{k}-2\right) \varepsilon^{1 / 2^{k}}\right\}, \\
S & :=\{u \in V \mid S(u)>2 \varepsilon n\},
\end{aligned}
$$

where $H_{u, v}$ is the sameness $(k-1)$-graph of $H$ with respect to $u$ and $v$ defined previously (i.e., $\mu_{H_{u, v}}(X)=-\mu_{H}(\{u\} \cup X) \mu_{H}(\{v\} \cup X)$ for $\left.X \in\left(\begin{array}{c}V \\ k-1\end{array}\right)\right)$. If $|S| \leq 2 \varepsilon n$ then

$$
\begin{aligned}
\operatorname{dev} H & =\frac{1}{n^{2}} \sum_{u, v} \operatorname{dev} H_{u, v} \quad \text { by Facts } 4.0 \text { and } 4.3 \\
& \leq \frac{1}{n^{2}}\left\{|S| \cdot n+\left(4^{k}-2\right) \varepsilon^{1 / 2^{k}} \cdot n^{2}\right\} \leq 4^{k} \varepsilon^{1 / 2^{k}}
\end{aligned}
$$

as required. Thus, we may assume $|S|>2 \varepsilon n$. Fix $u \in S$. For each $v \in S(u)$, the induction hypothesis implies there exists a $(k-2)$-graph $G(u, v)$ on $V$ satisfying

$$
\left|e\left(H_{u, v}, G(u, v)\right)-e\left(\bar{H}_{u, v}, G(u, v)\right)\right|>6 \delta^{2} n^{k-1}
$$

where $\delta^{2}=4 \varepsilon$. Thus, there is a subset $S^{\prime}(u)$ of $S(u)$ with $\left|S^{\prime}(u)\right|=\varepsilon n$ so that either:

(a) $\left.e\left(H_{u, v}, G(u, v)\right) \geq \frac{1}{2} e\left(\left(\begin{array}{c}V \\ k-1\end{array}\right)\right), G(u, v)\right)+3 \delta^{2} n^{k-1}$ for all $v \in S^{\prime}(u)$; or

(b) $\left.e\left(H_{u, v}, G(u, v)\right) \leq \frac{1}{2} e\left(\left(\begin{array}{c}V \\ k-1\end{array}\right)\right), G(u, v)\right)-3 \delta^{2} n^{k-1}$ for all $v \in S^{\prime}(u)$.

We will just treat case (a); the argument for case (b) is very similar and is omitted. We begin by defining the following $(k-1)$-graphs on $V$.

$$
\begin{aligned}
E\left(H_{u}^{\prime}\right) & =E(H(u)) \cap\left(\begin{array}{c}
V \backslash S^{\prime}(u) \\
k-1
\end{array}\right), \\
E\left(H_{u}^{\prime \prime}\right) & =E(\overline{H(u)}) \cap\left(\begin{array}{c}
V \backslash S^{\prime}(u) \\
k-1
\end{array}\right), \\
E\left(G^{\prime}\right) & =\left\{Y \cup\{v\} \mid Y \in E(G(u, v)), v \in S^{\prime}(u)\right\} \cup E\left(H_{u}^{\prime}\right), \\
E\left(G^{\prime \prime}\right) & =\left\{Y \cup\{v\} \mid Y \in E(G(u, v)), v \in S^{\prime}(u)\right\} \cup E\left(H_{u}^{\prime \prime}\right) .
\end{aligned}
$$

Consider the sum

$$
\sigma(u):=\sum_{v \in S^{\prime}(u)} e\left(H_{u, v}, G(u, v)\right) .
$$

For each $X^{\prime} \in E\left(H, G^{\prime}\right)$ there are three possibilities:

(i) $\left|X^{\prime} \cap S^{\prime}(u)\right| \geq 2$. There are at most $\varepsilon^{2} n^{k}$ such $X^{\prime}$.

(ii) $\left|X^{\prime} \cap S^{\prime}(u)\right|=1$. In this case, $X^{\prime}=Y \cup\{v\}$ for some $v \in S^{\prime}(u)$, and so, $Y \in E\left(H_{u, v}, G(u, v)\right)$. 
(iii) $\left|X^{\prime} \cap S^{\prime}(u)\right|=0$. In this case, $X^{\prime} \in E\left(H, H_{u}^{\prime}\right)$.

A similar analysis applies to those $X^{\prime \prime} \in E\left(H, G^{\prime \prime}\right)$. Combining these observations, we obtain

$$
\begin{aligned}
& \sigma(u)= \sum_{v \in S^{\prime}(u)} e\left(H_{u, v}, G(u, v)\right) \\
&=(k-1) ! \sum_{v \in S^{\prime}(u)} \mid\left\{Y\left|Y \in E\left(H_{u, v}, G(u, v)\right\}\right|\right. \\
&=(k-1) ! \sum_{v \in S^{\prime}(u)}\left|\left\{Y \cup\{v\} \mid Y \in E\left(H_{u, v}, G(u, v)\right)\right\}\right| \\
& \leq(k-1) ! \mid\left\{X \in\left(\begin{array}{l}
V \\
k
\end{array}\right)|| X \cap S^{\prime}(u) \mid=1, \quad X=Y \cup\{v\},\right. \\
&\left.\qquad Y \in E\left(H_{u, v}, G(u, v)\right)\right\} \mid+\varepsilon^{2} n^{k} \\
&=(k-1) ! \mid\left\{X|| X \cap S^{\prime}(u) \mid=1, \quad X=Y \cup\{v\}, X \in E(H),\right. \\
& \quad \quad Y \in\left(H_{u, v}, G(u, v)\right\} \mid \\
& \leq e\left(H, G^{\prime}\right)-e\left(H, H_{u}^{\prime}\right)+e\left(\bar{H}, G^{\prime \prime}\right)-e\left(\bar{H}, H_{u}^{\prime \prime}\right)+\varepsilon^{2} n^{k} .
\end{aligned}
$$

Now, if we apply the induction hypothesis to the $(k-1)$-graphs $H_{u}^{\prime}, H_{u}^{\prime \prime}, G^{\prime}$, and $G^{\prime \prime}$, we have

$$
\begin{aligned}
\left|e\left(H, H_{u}^{\prime}\right)-e\left(\bar{H}, H_{u}^{\prime}\right)\right| & <2 \varepsilon^{2} n^{k}, \\
\left|e\left(H, H_{n}^{\prime \prime}\right)-e\left(\bar{H}, H_{u}^{\prime \prime}\right)\right| & <2 \varepsilon^{2} n^{k}, \\
\left|e\left(H, G^{\prime}\right)-e\left(\bar{H}, G^{\prime}\right)\right| & <2 \varepsilon^{2} n^{k}, \\
\left|e\left(H, G^{\prime \prime}\right)-e\left(\bar{H}, G^{\prime \prime}\right)\right| & <2 \varepsilon^{2} n^{k} .
\end{aligned}
$$

Consequently, from (8.7)

$$
\begin{aligned}
\sigma(u) \leq & \frac{1}{2} e\left(\left(\begin{array}{l}
V \\
k
\end{array}\right), G^{\prime}\right)-\frac{1}{2} e\left(\left(\begin{array}{l}
V \\
k
\end{array}\right), H_{u}^{\prime}\right) \\
& +\frac{1}{2} e\left(\left(\begin{array}{l}
V \\
k
\end{array}\right), G^{\prime \prime}\right)-\frac{1}{2} e\left(\left(\begin{array}{l}
V \\
k
\end{array}\right), H_{u}^{\prime \prime}\right)+5 \varepsilon^{2} n^{k} .
\end{aligned}
$$

On the other hand, by (i) we see

$$
\begin{aligned}
\sigma(u) \geq & \frac{1}{2} \sum_{v \in S^{\prime}(u)} e\left(\left(\begin{array}{c}
V \\
k-1
\end{array}\right), G(u, v)\right)+3 \delta^{2} n^{k-1}|S(u)| \\
\geq & \frac{1}{2} e\left(\left(\begin{array}{l}
V \\
k
\end{array}\right), G^{\prime}\right)-\frac{1}{2} e\left(\left(\begin{array}{l}
V \\
k
\end{array}\right), H_{u}^{\prime}\right) \\
& +\frac{1}{2} e\left(\left(\begin{array}{l}
V \\
k
\end{array}\right), G^{\prime \prime}\right)-\frac{1}{2} e\left(\left(\begin{array}{l}
V \\
k
\end{array}\right), H_{u}^{\prime \prime}\right)-5 \varepsilon^{2} n^{k}+3 \delta^{2} \varepsilon n^{k} .
\end{aligned}
$$


However, (8.8) and (8.9) imply $\delta^{2} \leq 10 \varepsilon / 3$, which contradicts the assumption that $\delta^{2}=4 \varepsilon$.

This completes the induction step and Theorem 8.1 is proved.

We point out here that it is possible to define, for any $l \leq k$, the $l$-discrepancy $\operatorname{disc}_{l} H$ of a $k$-graph $H$ on a vertex set $V$ of size $n$ by

$$
\operatorname{disc}_{l} H:=\frac{1}{n^{k}} \max _{G}|e(H, G)-e(\bar{H}, G)|,
$$

where $G$ ranges over all $(l-1)$-graphs on $V, E(H, G)=\left\{X \in E(H) \mid\left(\begin{array}{c}X \\ l-1\end{array}\right) \subset\right.$ $E(G)\}, e(H, G)=k !|E(H, G)|$, etc. For this more general concept, the analogue to Theorem 8.1 holds.

Theorem 8.2. For $2 \leq l \leq k$,

(i) $\operatorname{disc}_{l} H \leq\left(\operatorname{dev}_{l} H\right)^{2^{-l}}$;

(ii) $\operatorname{dev}_{l} H \leq 4^{l}\left(\operatorname{disc}_{l} H\right)^{2^{-l}}$.

The proof of Theorem 8.2 is similar to that of Theorem 8.1 and is given in Chung [C90], which in fact includes a much fuller discussion of $l$-deviation and $l$-discrepancy, and the applications of these ideas to communication complexity (cf. [BNS89]).

\section{GRAPHS}

Certainly the most commonly occurring $k$-graphs are just (ordinary) graphs. While many of the known results relating the deviation of a graph $G$ to other structural invariants follow immediately from the preceding results by specializing $k$ to be 2 , there are other useful properties of graphs that are not easily generalized to larger values of $k$. In this section, we discuss some of these.

To begin with, for each graph $G=\left(V, \mu_{G}\right)$ on vertices, we can define (as before) an $n \times n$ symmetric matrix $A=A(G)=(a(x, y))_{x, y \in V}$, called the adjacency matrix of $G$, as follows:

$$
a(x, y)= \begin{cases}1 & \text { if }\{x, y\} \text { is an edge of } G, \\ 0 & \text { otherwise }\end{cases}
$$

Since $A(G)$ is real symmetric, its eigenvalues $\lambda_{i}=\lambda_{i}(G), 1 \leq i \leq n$, are real. We label them so that

$$
\left|\lambda_{1}\right| \geq\left|\lambda_{2}\right| \geq \cdots \geq\left|\lambda_{n}\right| \text {. }
$$

It is well known (e.g., see [CDS80, CDGT88]) that many structural properties of $G$ are controlled by the behavior of the eigenvalues of $A(G)$. Random graphs $G_{1 / 2}(n)$ are known [FK81] a.a. to have

$$
\lambda_{1}=(1+o(1)) n / 2, \quad \lambda_{2}=O\left(n^{1 / 2}\right), \quad n \rightarrow \infty .
$$

In [CGW89], it is shown that the conjunction of the following three properties is equivalent to a family of graphs $G=G(n)$ being quasi-random, i.e., 
equivalent to having $\operatorname{dev} G=o(1), n \rightarrow \infty$ :

(a) $G$ has $(1+o(1)) n^{2} / 4$ edges;

(b) $\lambda_{1}(G)=(1+o(1)) n / 2$;

(c) $\lambda_{2}(G)=o(n)$.

The following theorem is a more quantitative form of this result, which allows it to be applied to individual graphs, rather than just having it apply asymptotically to a family of graphs.

Theorem 9.1. For any graph $G=G(n)=\left(V, \mu_{G}\right)$;

(i) $\left|\sum_{x, y} \mu_{G}(x, y)\right| \leq n^{2}(\operatorname{dev} G)^{1 / 4}$;

(ii) $\left|\lambda_{1}(G)-\frac{n}{2}\right| \leq \frac{n}{2}(\operatorname{dev} G)^{1 / 4}$;

(iii) $\left|\lambda_{2}(G)\right| \leq n(\operatorname{dev} G)^{1 / 16}$.

Proof. Let $e$ denote the number of edges of $G$, let $\bar{e}:=n^{2} / 2-e$, and let $A=A(G)$ denote the adjacency matrix of $G$.

First observe that (i) follows immediately from Fact 4.9 (applying it twice), since

$$
\operatorname{dev}_{0} G=\frac{1}{n^{2}} \sum_{x, y} \mu_{G}(x, y)=1-\frac{4 e}{n^{2}} .
$$

The proof of (ii) is slightly more complex. To begin with we have (by RayleighRitz)

$$
\lambda_{1}=\sup _{v} \frac{\langle v, A v\rangle}{\langle v, v\rangle} \geq \frac{\langle\overline{1}, A \overline{1}\rangle}{n}=\frac{2 e}{n}
$$

where $\overline{1}$ denotes the vector of all 1's. Therefore,

$$
\frac{n}{2}-\lambda_{1} \leq \frac{n}{2}-\frac{2 e}{n}
$$

Define the matrix $M=(m(x, y))_{x, y \in V}=J-2 A$, where $J$ denotes the $n \times n$ matrix of all 1's; thus, $m(x, y)=\mu_{G}(x, y)$. Also, set

$$
\begin{gathered}
d(x):=\left|\left\{y \in V \mid \mu_{G}(x, y)=-1\right\}\right|, \quad \bar{d}(x):=n-d(x), \\
s(x, y):=\left|\left\{z \in V \mid \mu_{G}(x, z)=\mu_{G}(y, z)\right\}\right|, \quad \bar{s}(x, y):=n-s(x, y) .
\end{gathered}
$$

Since

$$
2|e-\bar{e}|=\left|\sum_{x, y} m(x, y)\right| \leq\left|\sum_{x}(d(x)-\bar{d}(x))\right| \leq \sum_{x}|d(x)-\bar{d}(x)|,
$$


then

$$
\begin{aligned}
n^{4} \operatorname{dev} G & =\sum_{x, y}(s(x, y)-\bar{s}(x, y))^{2} \\
& \geq \frac{1}{n^{2}}\left(\sum_{x, y}|s(x, y)-\bar{s}(x, y)|\right)^{2} \\
& \geq \frac{1}{n^{2}}\left(\sum_{x, y}(s(x, y)-\bar{s}(x, y))\right)^{2} \\
& \geq \frac{1}{n^{2}}\left(\sum_{x}(d(x)-\bar{d}(x))^{2}\right)^{2} \\
& \geq \frac{1}{n^{2}}\left(\frac{1}{n}\left(\sum_{x}|d(x)-\bar{d}(x)|\right)^{2}\right)^{2} \\
& \geq \frac{16}{n^{4}}(e-\bar{e})^{4} .
\end{aligned}
$$

Since $M=J-2 A$ and the $\lambda_{i}$ are real, then

$$
16 \lambda_{1}^{4} \leq 16 \operatorname{Tr}\left(A^{4}\right)=\operatorname{Tr}(J-M)^{4}
$$

where $\operatorname{Tr}$ denotes the trace function. By the additivity of $\operatorname{Tr}$, we can upper bound $\operatorname{Tr}(J-M)^{4}$ by bounding the various terms we obtain by expanding $(J-M)^{4}$. To do this, we note the following inequalities:

$$
\begin{aligned}
\operatorname{Tr}\left(J^{4}\right) & =n^{4}, \\
\operatorname{Tr}\left(J^{3} M\right) & =\operatorname{Tr}\left(J^{2} M J\right)=\operatorname{Tr}\left(J M J^{2}\right)=\operatorname{Tr}\left(M J^{3}\right) \leq n^{4}(\operatorname{dev} G)^{1 / 4}, \\
\operatorname{Tr}\left(M^{2} J^{2}\right) & =\operatorname{Tr}\left(J^{2} M^{2}\right)=\operatorname{Tr}\left(J M^{2} J\right) \leq n \sum_{x, y}(s(x, y)-\bar{s}(x, y)) \leq n^{4}(\operatorname{dev} G)^{1 / 2}, \\
\operatorname{Tr}(M J M J) & =\operatorname{Tr}(J M J M) \leq\left(\sum_{x}(d(x)-\bar{d}(x))^{2} \leq n^{4}(\operatorname{dev} G)^{1 / 2}\right. \\
\operatorname{Tr}\left(M J^{2} M\right) & \leq n \sum_{x}(d(x)-\bar{d}(x))^{2} \leq n^{4}(\operatorname{dev} G)^{1 / 2}, \\
\operatorname{Tr}\left(J M^{3}\right) & \leq \sum_{x, y}(d(x)-\bar{d}(x))(s(x, y)-\bar{s}(x, y)) \\
& \leq\left(\left(n_{z}(d(z)-\bar{d}(z))^{2}\left(\sum_{x, y}(s(x, y)-\bar{s}(x, y))^{2}\right)\right)^{1 / 2}\right. \\
& \leq\left(n^{4}(\operatorname{dev} G)^{1 / 2} \cdot n^{4}(\operatorname{dev} G)\right)^{1 / 2} \leq n^{4}(\operatorname{dev} G)^{3 / 4}, \\
\operatorname{Tr}\left(M J M^{2}\right) & =\operatorname{Tr}\left(M^{2} J M\right) \leq \sum_{x, y}(d(x)-\bar{d}(x))(s(x, y)-\bar{s}(x, y)) \\
& \leq n^{4}(\operatorname{dev} G)^{3 / 4},
\end{aligned}
$$




$$
\begin{aligned}
\operatorname{Tr}\left(M^{3} J\right) & \leq n^{4}(\operatorname{dev} G)^{3 / 4}, \\
\operatorname{Tr}\left(M^{4}\right) & =n^{4} \operatorname{dev} G .
\end{aligned}
$$

Therefore,

$$
\begin{aligned}
\operatorname{Tr}(J-M)^{4} \leq & n^{4}+4 n^{4}(\operatorname{dev} G)^{1 / 4}+6 n^{4}(\operatorname{dev} G)^{1 / 2} \\
& +4 n^{4}(\operatorname{dev} G)^{3 / 4}+n^{4} \operatorname{dev} G \\
= & n^{4}\left(1+(\operatorname{dev} G)^{1 / 4}\right)^{4}
\end{aligned}
$$

which implies

$$
\lambda_{1}^{4} \leq\left(\frac{n}{2}\right)^{4}\left(1+(\operatorname{dev} G)^{1 / 4}\right)^{4}
$$

i.e.,

$$
\lambda_{1} \leq\left(\frac{n}{2}\right)\left(1+(\operatorname{dev} G)^{1 / 4}\right)
$$

Thus, by (9.3), (9.4), and (9.5) we have

$$
\left|\lambda_{1}-\frac{n}{2}\right| \leq \frac{n}{2}(\operatorname{dev} G)^{1 / 4}
$$

as required for (ii).

Finally, to prove (iii) we have

$$
\begin{aligned}
\lambda_{2}^{4} & \leq \operatorname{Tr}\left(A^{4}\right)-\lambda_{1}^{4} \\
& \leq \frac{1}{16} \operatorname{Tr}(J-M)^{4}-\lambda_{1}^{4} \\
& \leq \frac{1}{16} n^{4}\left(1+(\operatorname{dev} G)^{1 / 4}\right)^{4}-\left(\frac{n}{2}\left(1-(\operatorname{dev} G)^{1 / 4}\right)\right)^{4} \\
& \leq\left(\frac{n}{2}\right)^{4} \cdot 8(\operatorname{dev} G)^{1 / 4}\left(1+(\operatorname{dev} G)^{1 / 2}\right) \\
& \leq n^{4}(\operatorname{dev} G)^{1 / 4}
\end{aligned}
$$

i.e.,

$$
\left|\lambda_{2}\right| \leq n(\operatorname{dev} G)^{1 / 16}
$$

This completes the proof of Theorem 9.1.

In turns out that the $\square$-product described in $\S 3$ can be used to form arbitrarily large quasi-random graphs that are essentially optimal from the point of view of having $\lambda_{2}$ small. Here is an outline of the relevant facts. Let $G=G(n)$ be a graph with $\operatorname{dev} G<1$ (i.e., $G$ is not a complete bipartite graph). Let $A(G)$ be the adjacency matrix of $G$, and let $M=M(G)=J-2 A(G)$, where $J$ is the $n \times n$ matrix of all 1's. Thus, $M$ is symmetric with 1's on the diagonal. Let $\omega_{i}$, $1 \leq i \leq m$, denote the eigenvalues of $M$, ordered so that $\left|\omega_{1}\right| \geq\left|\omega_{2}\right| \geq \cdots \geq$ $\left|\omega_{n}\right|$. Then $G^{\square t}:=\square_{i=1}^{t} G$ has $M\left(G^{\square t}\right):=M^{(t)}=\bigotimes_{i=1}^{t} M$ (the ordinary tensor product). Since $\operatorname{dev} G<1$ then $\operatorname{dev}\left(G^{\square t}\right)=(\operatorname{dev} G)^{t}=o(1)$ as $t \rightarrow \infty$. Thus, $M^{(t)}$ has as an eigenvector $\overline{1}+\bar{\varepsilon}$ where $\overline{1}$ is the all 1 's vector of length $N=n^{t}$, 
and each component of $\bar{\varepsilon}$ is $O(1)$. This implies that the largest eigenvalue of $A^{(t)}:=A\left(G^{\square t}\right)$ is $(1+o(1)) N / 2$ while $\lambda_{2}^{(t)}$, the second largest eigenvalue of $A^{(t)}$ (in absolute value) is at most $\left(\frac{1}{2}+o(1)\right)\left|\omega_{1}\right|^{t}=\left(\frac{1}{2}+o(1)\right) N^{\log \left|\omega_{1}\right| / \log n}$. Now, it is well known (see [FK81]) that if a graph $H$ on $N$ vertices has all but $o(N)$ vertices with degrees $(1+o(1)) N / 2, N \rightarrow \infty$, then $\lambda_{2}(H)$, its second largest eigenvalue, must satisfy $\lambda_{2}(H)>c N^{1 / 2}$ for some $c>0$. Could our product graphs $G^{\square t}$ meet this bound? They could, but only if $\log \left|\omega_{1}\right| / \log n=1 / 2$, i.e., $\left|\omega_{1}\right|=\sqrt{n}$, for the starting matrix $M$. However, since

$$
\operatorname{trace}\left(M^{T} M\right)=n^{2}=\sum_{i=1}^{n} \omega_{i}^{2},
$$

then the only way $\left|\omega_{1}\right|$ can equal $\sqrt{n}$ is for all $\omega_{i}= \pm \sqrt{n}$ (as observed by $\mathrm{L}$. Lovász [L89]). This implies that if we set $U$ to be the (unitary) matrix formed by the eigenvectors $\bar{e}_{i}$, then

$$
\left(U^{T} M^{T}\right)(M U)=U^{-1} M^{T} M U=n I,
$$

i.e., $M^{T} M=n I$, which just means that $M$ is a Hadamard matrix. Since $\operatorname{Tr}(M)=n=\sum_{i=1}^{n} \omega_{i}$ and each $\omega_{i}= \pm \sqrt{n}$, then $n$ must be a perfect square. The smallest nontrivial example of this is given by the matrix

$$
M_{4}=\left[\begin{array}{cccc}
1 & 1 & 1 & 1 \\
1 & 1 & -1 & -1 \\
1 & -1 & 1 & -1 \\
1 & -1 & -1 & 1
\end{array}\right]
$$

with corresponding 4-vertex graph

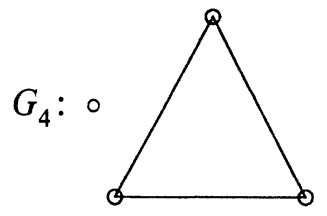

Of course, there are other ways to generate symmetric Hadamard matrices (with diagonal 1) besides taking tensor products (see [Wa88]). However, it is interesting that the simple $\square$-product can produce graphs with such good $\lambda_{2}$ behavior.

Of course in principle all of the asymptotic results in [CGW89] and [CG90(a)] have explicit versions (i.e., not involving $o(1)$ ). We give Theorem 9.1 as an example of just how such a translation can be made in this case. The reverse direction, bounding $\operatorname{dev} G$ in terms of the maxima of the three quantities $\left|e-n^{2} / 4\right|,\left|\lambda_{1}-n / 2\right|$ and $\left|\lambda_{2}\right|$, we leave as an interesting exercise for the reader. We mention one more such translation since it involves one of the most innocuous sounding conditions for quasi-randomness. Here, $C_{4}$ denotes a 4-cycle, i.e., the graph with vertices $\{a, b, c, d\}$ and edges $\{a, b\},\{b, c\}$, $\{c, d\}$, and $\{d, a\}$. 
Theorem 9.2. Let $\{G(n)\}$ be a family of graphs where $G(n)$ has $n$ vertices and $e(G(n))$ edges. Then

if and only if

$$
\operatorname{dev} G(n)=o(1)
$$

$e(G(n)) \geq(1+o(1)) n^{2} / 4$ and $\#\left\{C_{4} \subset G(n)\right\} \leq(1+o(1)) n^{2} / 16, \quad n \rightarrow \infty$.

An explicit form of this result (which can be proved along the lines given in the proof of Theorem 9.1) is

Theorem 9.3. Let $G$ be any graph with $n$ vertices and $e(G)$ edges. Then,

(i) $e(G) \geq\left(n^{2} / 4\right)\left(1-(\operatorname{dev} G)^{1 / 4}\right)$;

(ii) $\#\left\{C_{4} \subset G\right\} \leq\left(n^{4} / 16\right)\left(1+(\operatorname{dev} G)^{1 / 4}\right)^{4}$. Also,

(iii) $e(G) \geq\left(\frac{1}{4}-\alpha\right) n^{2}$ and $\#\left\{C_{4} \subset G\right\} \leq\left(\frac{1}{4}+\alpha\right)^{2} n^{4}$ implies $\operatorname{dev} G \leq$ $8 \alpha(33-26 \alpha)$.

It would be interesting to know what the sharpest results of this form are. (A recent paper of Spencer and Tetali [ST(a)] also deals with quantitative aspects of quasi-randomness for graphs.)

A curious singularity occurs for graphs in connection with Facts 4.7 and 4.8. It follows from these results that for any $0<\alpha<1, \operatorname{dev} G(n)=o(1)$ if and only if for all $X \subset V$ with $|X|=(1+o(1)) \alpha n, G[X]$ has $(1+o(1)) \alpha^{2} n^{2} / 4$ edges. In particular, this implies that $e(X, \bar{X})$, defined to be the number of edges in $G$ that hit both $X$ and $\bar{X}$, satisfies

$$
e(X, \bar{X})=(1+o(1)) \alpha(1-\alpha) n^{2} / 2 \text {. }
$$

A natural question is whether the converse holds, that is, if (9.7) holds for all $X \subset V$ with $|X|=(1+o(1)) \alpha n$ then $\operatorname{dev} G(n)=o(1)$ (i.e., $\{G(n)\}$ is quasi-random).

Any such hopes, however, are shattered by the following obstruction. Let $H$ be a graph with vertex set $A \cup B$, with $A$ and $B$ disjoint and $|A|=|B|=n / 2$. The edge set of $H$ will consist of the pairs $\left(\begin{array}{l}A \\ 2\end{array}\right)$ together with a random bipartite graph between $A$ and $B$ (i.e., each edge $\{a, b\}$ is chosen independently with probability $1 / 2)$. Then it is not hard to see that for almost all $H$, every set $X \subset A \cup B$ of size $n / 2$ spans $n^{2} / 8+O(n)$ edges. However, a simple calculation shows that $\operatorname{dev} H=\frac{1}{2}+O(1)$.

What is surprising, however, is that $1 / 2$ is the only value of $\alpha$ for which the converse fails to hold. We outline a proof of this fact.

Theorem 9.4. Let $0<\alpha<1 / 2$ and suppose $G=G(n)=(V, \mu)$ satisfies (9.7) for all $X \subset V$ with $|X|=(1+o(1)) \alpha n$. Then $\operatorname{dev} G=o(1), n \rightarrow \infty$.

Proof. To begin with, we define for integers $r$ and $t$ with $3 \leq r<t / 2$ the matrix $M=M_{r, t}=(M(I, e))$, where $I$ ranges over all $\left(\begin{array}{c}{[t]} \\ r\end{array}\right)$, the set of $r$ element subsets of $[t]:=\{1,2, \ldots, t\}, e$ ranges over $\left(\begin{array}{c}{[t]} \\ 2\end{array}\right)$, and

$$
M(I, e)= \begin{cases}1 & \text { if }|e \cap I|=1 \\ 0 & \text { otherwise }\end{cases}
$$


We can think of forming a complete graph $K_{t}$ on $[t]$, and, for each complete bipartite graph $K(I, \bar{I})$ (on vertex sets $I$ and $\bar{I}:=[t] \backslash I$ ) and each edge $e$, letting $M(I, e)$ indicate which $e$ are edges of $K(I, \bar{I})$. A related, but somewhat more complicated, matrix $M^{*}=\left(M^{*}(e, I)\right)$ is given by

$$
M^{*}(e, I)= \begin{cases}-(r-1)(r(t-2 r)+2(r-1)) & \text { if }|e \cap I|=0, \\ (r-1)(t-r-1)(t-2 r) & \text { if }|e \cap I|=1, \\ -(t-r-1)((t-r)(t-2 r)-2(t-r-1)) & \text { if }|e \cap I|=2,\end{cases}
$$

where, as in $M, I \in\left(\begin{array}{c}{[t]} \\ r\end{array}\right)$ and $e \in\left(\begin{array}{c}{[t]} \\ 2\end{array}\right)$. In particular, $M$ is $\left(\begin{array}{c}t \\ r\end{array}\right) \times\left(\begin{array}{l}t \\ 2\end{array}\right)$ and $M^{*}$ is $\left(\begin{array}{l}t \\ 2\end{array}\right) \times\left(\begin{array}{l}t \\ r\end{array}\right)$.

The two matrices $M$ and $M^{*}$ are related by

$$
M^{*} M=2(t-2 r) \frac{(t-2) !}{(r-2) !(t-r-2) !} I_{\left(\begin{array}{l}
t \\
2
\end{array}\right)}
$$

where $I_{\left(\begin{array}{l}t \\ 2\end{array}\right)}$ is the identity matrix of size $\left(\begin{array}{l}t \\ 2\end{array}\right)$. Equation (9.8) follows by direct computation using the definitions of $M$ and $M^{*}$. Thus, $M^{*}$ is a (scalar multiple of a) left inverse of $M$, and it follows in particular that $M$ has full rank, i.e., rank equal to $\left(\begin{array}{l}t \\ 2\end{array}\right)$. We remark that for $t=2 r$, the matrix $M_{r, t}=$ $M_{r, 2 r}$ only has rank $\left(\begin{array}{c}2 r-1 \\ 2\end{array}\right)$. This turns out to be the underlying reason for the special behavior of the value $\alpha=1 / 2$.

Now, consider the property $Q^{(\alpha)}(\varepsilon)$ for $\varepsilon>0, \alpha<1 / 2$, defined by:

$$
\begin{aligned}
& Q^{(\alpha)}(\varepsilon): \text { If } X \subset V \text { with }|X-\alpha n|<\varepsilon n \text { then } \mid e(X, \bar{X})- \\
& \frac{1}{2} \alpha(1-\alpha) n^{2} \mid<\varepsilon n^{2} \text { for } n>n_{0}(\varepsilon) .
\end{aligned}
$$

We want to apply $Q^{(\alpha)}(\alpha)$ to $G=G(n)$ in the following way. Let $t$ be large (but fixed) and assume for ease of exposition that $n=t m$ for some integer $m$. Partition the vertex set $V$ of $G$ into disjoint sets $C_{1}, C_{2}, \ldots, C_{t}$, each of size $m$, and define

$$
\rho_{i j}:=\frac{1}{m^{2}} e\left(C_{i}, C_{j}\right), \quad 1 \leq i<j \leq t,
$$

where $e\left(C_{i}, C_{j}\right)$ denotes the number of edges of $G$ between $C_{i}$ and $C_{j}$. We can associate with this construction a weighted complete graph $K_{t}$ on $[t]$, with the edge $e=\{i, j\}$ of $K_{t}$ receiving the weight $\rho(e)=\rho_{i j}$.

We now fix $r$ with $3 \leq r<t / 2$ so that $\beta:=r / t$ is close to $\alpha$ (we will be more precise later). We first apply $Q^{(\beta)}(\varepsilon)$ to $G$. This then implies that for each $I \subset\left(\begin{array}{c}(t]) \\ r\end{array}\right)$, if we form $X=\bigcup_{i \in I} C_{i}$ then the number $e(X, \bar{X})=c(I)$ of crossing edges, which is just

$$
c(I)=\sum_{\substack{i \in I \\ j \in \bar{I}}} e\left(C_{i}, C_{j}\right)=m^{2} \sum_{\substack{i \in I \\ j \in \bar{I}}} \rho_{i j}
$$

satisfies

$$
m^{2} M \bar{\rho}=\bar{c},
$$


where $\bar{\rho}=[\rho(e)]_{e \in\left(\begin{array}{c}{[t]} \\ 2\end{array}\right)}$ and $\bar{c}=[c(I)]_{I \in\left(\begin{array}{c}{[t]} \\ r\end{array}\right)}$ are column vectors. By $Q^{(\beta)}(\varepsilon)$, we know

$$
c(I)=\left(\frac{1}{2} \beta(1-\beta)+\varepsilon(I)\right) n^{2},
$$

where $|\varepsilon(I)|<\varepsilon, I \in\left(\begin{array}{c}{[t]} \\ r\end{array}\right)$.

Now, we invert $(9.10)$ by left-multiplying by $M^{*}$ to get

$$
m^{2} M^{*} M \bar{\rho}=2(t-2 r) \frac{(t-2) !}{(r-2) !(t-r-2) !} \bar{\rho}=M^{*} \bar{c} .
$$

However, direct computation shows that

$$
M^{*} \bar{I}=\frac{2(t-2 r)(t-2) !}{r(t-r)(r-2) !(t-r-2) !} \overline{1}
$$

where $\overline{1}$ denotes a column vector of all 1's. Thus, we obtain from (9.11), (9.12), and (9.13)

$$
\left|\rho(e)-\frac{1}{2} \beta(1-\beta) n^{2}\right|<\varepsilon n^{2}
$$

for each $e \in\left(\begin{array}{c}{[t]} \\ 2\end{array}\right)$ and $n>n_{0}(\varepsilon)$. This means that all the "edge densities" $\rho_{i j}$ between the various clusters $C_{i}$ and $C_{j}$ in $G$ are very close to what is expected. Of course, to apply $Q^{(\alpha)}$ rather than $Q^{(\beta)}$, we choose a sufficiently close rational approximation $\beta=r / t$ to $\alpha$. It then finally follows that any $n / 2$ points of $G$ span $\frac{1}{8} \alpha(1-\alpha) n^{2}+o\left(n^{2}\right)$ edges, which in turn implies quasi-randomness, i.e., $\operatorname{dev} G=o(1)$. This argument works for $\alpha \neq 1 / 2$ and fails for $\alpha=1 / 2$ precisely because the matrix $M_{r, t}$ has full rank $\left(\begin{array}{l}t \\ 2\end{array}\right)$ for $2 \leq r \leq t-2, r \neq t / 2$, but only has rank $\left(\begin{array}{c}t-1 \\ 2\end{array}\right)$ when $r=t / 2$ (which corresponds to $\alpha=1 / 2$ ).

A fuller discussion (and a completely different proof) of this result is given in $[C G(a)]$. We do not know at present what the corresponding results are for $k>2$. The first case would be: Suppose $G=G(n)=(V, \mu)$ is a 3-graph so that for any partition $V=A \cup B \cup C$ with $|A|=|B|=|C|=n / 3$, the number $e(A, B, C)$ of edges of $G$ of the form $\{a, b, c\}$ and $a \in A, b \in B, c \in C$ satisfies

$$
e(A, B, C)=(1+o(1)) n^{3} / 54 .
$$

Does this imply $\operatorname{dev}(G)=o(1)$ as $n \rightarrow \infty$ ?

We conclude this section by pointing out that as soon as a family of graphs $G(n)$ fails to satisfy one of the quasi-random properties, then in fact all quasirandom properties must fail for $G(n)$. Relatively little is known quantitatively about this phenomenon. In [CG90(b)], the following is proved.

Theorem 9.5. Let $H(t)$ be an arbitrary fixed graph on $t$ vertices, and suppose that $\#\{H(t)<G(n)\}=0$ for a family of graphs $G(n), n \rightarrow \infty$. Then there exists $S \subset V(G(n))$ with $|S|=\lfloor n / 2\rfloor$ such that $\left|e(G[S])-n^{2} / 16\right|>2^{-\left(2 t^{2}+27\right)} n^{2}$ for $n \geq n_{0}(t)$. 
It is not whether a substantially sharper bound applies (e.g., of the form $2^{-\alpha t} n^{2}$ ), and just how the "truth" depends on the structure of the excluded graph $H(t)$.

\section{TOURNAMENTS}

In this section we show how some of the preceding ideas can be applied to the most commonly occurring directed graphs, namely, tournaments (e.g., see [M68]). We will not include all of the details (which can be found in [CG(c)] but rather discuss the basic results and show how they connect to ordinary graphs.

A tournament $T=\left(N, \mu_{T}\right)$ consists of a set $N=N(T)$, called the nodes of $T$, together with an (antisymmetric) function $\mu_{T}: N^{2} \rightarrow\{1,-1\}$. Thus, for $x \neq y$ in $N, \mu_{T}(x, y)=-\mu_{T}(y, x)$. By convention, $\mu_{T}(x, x)=1$ for all $x \in N$. The pairs $(x, y) \in \mu_{T}^{-1}(-1):=A(T)$ are called the $\operatorname{arcs}$ of $T$. As usual, $T(n)$ will denote a tournament on $n$ nodes. Define $n d^{-}(v)$ for a node $v$ of $T$ to be $\left\{u \in N \mid \mu_{T}(u, v)=-1\right\}$; similarly, define $n d^{+}(v):=\{u \in$ $\left.N \mid \mu_{T}(v, u)=-1\right\}$. The indegree $d^{-}(v)$ and outdegree $d^{+}(v)$ of $v$ are defined by

$$
d^{-}(v):=\left|n d^{-}(v)\right|, \quad d^{+}(v):=\left|n d^{+}(v)\right| .
$$

For $v \in N, X \subset N$, we let

$$
d^{-}(v, X):=\left|n d^{-}(v) \cap X\right|, \quad d^{+}(v, X):=\left|n d^{+}(v) \cap X\right| .
$$

Also, for $X, X^{\prime} \subset N$, define

$$
d^{-}\left(X, X^{\prime}\right):=\sum_{v \in X} d^{-}\left(v, X^{\prime}\right), \quad d^{+}\left(X, X^{\prime}\right):=\sum_{v \in X} d^{+}\left(v, X^{\prime}\right) .
$$

An ordering of $T=T(n)$ is a 1-to-1 mapping $\pi: N \rightarrow[n]=\{1,2, \ldots, n\}$. An arc $(u, v)$ is said to be $\pi$-increasing if $\pi(u)<\pi(v)$; otherwise we say that $(u, v)$ is $\pi$-decreasing. The undirected graph $T_{\pi}^{+}$on $N$ is formed by creating for each $\pi$-increasing arc $(u, v)$ of $T$ under the ordering $\pi$ an (undirected) edge $\{u, v\}$ of $T_{\pi}^{+}$.

For two nodes $u, v \in N$, the sameness $\operatorname{set} S(u, v)$ is defined by

$$
S(u, v):=\left\{z \in N \mid \mu_{T}(u, z)=\mu_{T}(v, z)\right\},
$$

and we let $s(u, v):=|S(u, v)|$.

If $T^{\prime}=\left(N^{\prime}, A^{\prime}\right)$ is a given tournament (or more generally, a directed graph), we let $\#\left\{T^{\prime}<T\right\}$ denote the number of labelled occurrences of $T^{\prime}$ as an induced subtournament (or sub-digraph) of $T$. In other words,

$$
\#\left\{T^{\prime}<T\right\}:=\left|\left\{\lambda: N^{\prime} \rightarrow N \mid T\left[\lambda\left(N^{\prime}\right)\right] \cong T^{\prime}\right\}\right|
$$

where $\cong$ denotes the obvious tournament isomorphism. Finally, we define a structure analogous to EPO's in the case of graphs. We call a sequence $\left(v_{0}, v_{1}, v_{2}, v_{3}\right)$ an even 4-cycle (E4C) if

$$
\mu_{T}\left(v_{0}, v_{1}\right) \mu_{T}\left(v_{1}, v_{2}\right) \mu_{T}\left(v_{2}, v_{3}\right) \mu_{T}\left(v_{3}, v_{0}\right)=1 .
$$


We let $\#\{E 4 C \subset T\}$ denote the number of (labelled) E4C's in $T$.

As in the case of graphs, we can define the deviation of $T=T(n)$ by

$$
\operatorname{dev} T:=\frac{1}{n^{4}} \sum_{v_{0}, v_{1}, v_{2}, v_{3}} \mu_{T}\left(v_{0}, v_{1}\right) \mu_{T}\left(v_{1}, v_{2}\right) \mu_{T}\left(v_{2}, v_{3}\right) \mu_{T}\left(v_{3}, v_{0}\right)
$$

We next state a collection of properties of a family of tournaments that are shared by almost all random tournaments $T_{1 / 2}(n), n \rightarrow \infty$. The essential content of our next theorem asserts the equivalence of all of these properties.

We only state them in their (weaker) asymptotic forms although we will indicate how they can be converted to "absolute" forms (i.e., with no occurrences of $o(1))$.

Theorem 10.1. For any family of tournaments $T=T(n)$, the following statements are equivalent as $n \rightarrow \infty$ :

(i) $\operatorname{dev} T=o(1)$;

(ii) For any fixed $x$, each tournament $T^{\prime}(s)$ on $s$ nodes satisfies

$$
\#\left\{T^{\prime}(s)<T\right\}=(1+o(1)) n^{s} 2^{-\left(\begin{array}{l}
s \\
2
\end{array}\right)}
$$

(iii) Each tournament $T^{\prime}(4)$ on 4 nodes satisfies

$$
\#\left\{T^{\prime}(4)<T\right\}=(1+o(1)) n^{4} / 64 ;
$$

(iv) $\#\{\mathrm{E} 4 \mathrm{C} \subset T\}=(1+o(1)) n^{4} / 2$;

(v) $\sum_{u, v \in N}|s(u, v)-n / 2|=o\left(n^{3}\right)$;

(vi) $\sum_{u, v \in N}||\left\{w \in N \mid \mu_{T}(u, w)=1=\mu_{T}(v, w)\right\}|-n / 4|=o\left(n^{3}\right)$;

(vii) For all $X \subset N, T^{\prime}=T[X]$ satisfies

$$
\sum_{v \in X}\left|d_{T^{\prime}}^{+}(v)-d_{T^{\prime}}^{-}(v)\right|=o\left(n^{2}\right)
$$

In this case we say that $T$ is almost balanced;

(viii) Every subtournament $T^{\prime}$ of $T$ on $\lfloor n / 2\rfloor$ nodes is almost balanced; have

(ix) For every partition of $N=X \cup Y$ with $|X|=\lfloor n / 2\rfloor,|Y|=\lceil n / 2\rceil$, we

$$
\sum_{v \in X}\left|d^{+}(v, Y)-d^{-}(v, Y)\right|=o\left(n^{2}\right)
$$

(x) For all $X, Y \subseteq N$,

$$
\sum_{v \in X}\left|d^{+}(v, Y)-d^{-}(v, Y)\right|=o\left(n^{2}\right)
$$

(xi) For every ordering $\pi$ of $T$,

$$
\mid\{u, v \in N \mid(u, v) \text { is } \pi \text {-increasing }\} \mid=(1+o(1)) n^{2} / 2 ;
$$

(xii) For every ordering $\pi$ of $T, \operatorname{dev} T_{\pi}^{+}=o(1)$;

(xiii) For some ordering $\pi$ of $T, \operatorname{dev} T_{\pi}^{+}=o(1)$. 
The last two properties relate quasi-randomness of tournaments to quasirandomness of graphs. However, there are several differences that should be noted.

On one hand, whereas $\operatorname{dev} G=1$ whenever $G$ is a complete bipartite graph, $\operatorname{dev} T$ is always bounded strictly below 1 . The exact value of $\rho:=\sup _{T} \operatorname{dev} T$ is not known, although it can be shown that $\frac{1}{3} \leq \rho \leq \frac{11}{12}$. (In $\S 11$, we characterize all $k$-graphs $H$ with $\operatorname{dev} H=1$.)

Further, it should be pointed out that the analogue to Theorem 9.4 does not hold for tournaments. To explain what we mean by this, consider the following set of properties for a family of tournaments $T(n)$ with node set $N$ :

(a) For the "cyclic" tournament $C_{3}$ with node set $\{1,2,3\}$ and arcs $(1,2)$, $(2,3),(3,1)$,

$$
\#\left\{C_{3}<T(n)\right\} \geq(1+o(1)) n^{3} / 8 ;
$$

(b) $T(n)$ is almost balanced, i.e.,

$$
\sum_{v \in N}\left|d_{T(n)}^{+}(v)-d_{T(n)}^{-}(v)\right|=o\left(n^{2}\right)
$$

(c) For every partition of $N=X \cup Y$,

$$
d^{+}(X, Y)-d^{-}(X, Y)=o\left(n^{2}\right)
$$

(d) For every partition of $N=X \cup Y$ with $|X|=\lfloor n / 2\rfloor,|Y|=\lceil n / 2\rceil$,

$$
d^{+}(X, Y)-d^{-}(X, Y)=o\left(n^{2}\right) \text {. }
$$

In $[C G(c)]$ it is shown that these four properties are equivalent. They are also strictly weaker than having $\operatorname{dev} T=o(1)$ as the following example shows.

Example. Let $T^{*}=T^{*}(n)$ have node set $X \cup Y \cup Z$ with $|X|=|Y|=|Z|=$ $n / 3$. Each of the subtournaments $T^{*}[X], T^{*}[Y], T^{*}[Z]$ will be random. The remaining arcs of $T^{*}$ are all the pairs $X \times Y, Y \times Z, Z \times X$. It is easily checked that (almost always) $T^{*}$ satisfies (a) (and therefore (b), (c), and (d)) but not any of the conditions in Theorem 10.1 , since $\operatorname{dev} T^{*} \rightarrow 2 / 9$ as $n \rightarrow \infty$. In particular, if we arbitrarily fix $\alpha \in(0,1)$ then for any $X \subset N$ with $|X|=$ $(1+o(1)) \alpha n$, we see by (c) that in $T^{*}$ there are $(1+o(1)) \alpha(1-\alpha) n^{2} / 2$ arcs from $X$ to $Y$. Thus, if we choose an ordering $\pi$ of $T^{*}$ so that all $(x, y), x \in X$, $y \in Y$ are $\pi$-increasing, then in the graph $T_{\pi}^{+}$, there are $(1+o(1)) \alpha(1-\alpha) n^{2} / 2$ edges between $X$ and $Y$.

As remarked earlier, all the tournament properties we have described can be formulated in absolute, as opposed to asymptotic, terms. For example, it can be shown (see $[\mathrm{CG}(\mathrm{c})]$ that:

$\left(\mathrm{v}^{\prime}\right) \sum_{u, v \in N}|s(u, v)-n / 2| \leq n^{3}(\operatorname{dev} T)^{1 / 2}$

(vii') For all $X \subset N, T^{\prime}=T[X]$ satisfies

$$
\sum_{v \in X}\left|d_{T^{\prime}}^{+}(v)-d_{T^{\prime}}^{-}(V)\right| \leq n^{2}(\operatorname{dev} T)^{1 / 4}
$$


$\left(\mathrm{xi}^{\prime}\right)$ For any ordering $\pi$ of $T$, ||$\{(u, v)$ is $\pi$-increasing $\}|-|\{(u, v)$ is $\pi$-decreasing $\}|| \leq 5 \sqrt{5} n^{2}(\operatorname{dev} T)^{1 / 8}$;

(xii') For every ordering $\pi$ of $T$, if $G=T_{\pi}^{+}$, the increasing arc graph of $T$ under $\pi$, then

$$
10^{-75}(\operatorname{dev} T)^{24} \leq \operatorname{dev} G \leq 40 \sqrt{300 \sqrt{5}}(\operatorname{dev} T)^{1 / 16}
$$

Of course, these bounds are rather crude and are only intended to illustrate the principle. It would be interesting to obtain sharp bounds for these various expressions (particularly $\left(\mathrm{xii}^{\prime}\right)$ ).

Properties (xii) and (xiii), linking graphs and tournaments, give us a potent new way for constructing large classes of graphs with small deviation from a single one with this property. Namely, suppose we start with a graph $G=$ $\left(V, \mu_{G}\right)$ where we assume $V=[n]=\{1,2, \ldots, n\}$. We can associate to $G$ a tournament $T=T_{G}=\left(V, \mu_{T}\right)$ by taking

$$
\mu_{T}(i, j)=\operatorname{sign}(j-i) \mu_{G}(\{i, j\}), \quad i \neq j .
$$

Thus, if id: $[n] \rightarrow[n]$ denotes the identity map then $G$ is just $T_{\text {id }}^{+}$. Now, let $\pi$ be an arbitrary ordering of $T$, and let $G_{\pi}:=T_{\pi}^{+}$. Applying (xii'), we obtain

$$
\operatorname{dev} G_{\pi}<2000(\operatorname{dev} G)^{1 / 384} \text {. }
$$

Thus, if $\operatorname{dev} G=o(1)$ then $\operatorname{dev} G_{\pi}=o(1)$ as $n \rightarrow \infty$. Of course, to go from $G$ to $G_{\pi}$ directly (avoiding intermediate tournaments), we simply permute the (ordered) vertex set with $\pi$, and interchange edges with nonedges for all pairs inverted by $\pi$. To the best of our knowledge, this transformation on graphs has not been treated before in the literature, so its properties are yet to be explored.

The preceding analysis can be carried out for ordered $k$-graph analogues of tournaments $T^{*}=\left(V, \mu^{*}\right)$. Here, $\mu^{*}: V^{k} \rightarrow\{1,-1\}$ so that for any permutation $\pi: V \rightarrow V$,

$$
\mu^{*}\left(\pi\left(x_{1}, \ldots, x_{k}\right)\right)=(-1)^{\operatorname{sign} \pi} \mu^{*}\left(x_{1}, \ldots, x_{k}\right),
$$

where $V^{\underline{k}}$ denotes $\left\{\left(x_{1}, \ldots, x_{k}\right) \in V^{k}: x_{i}\right.$ are distinct $\}$. We hope to return to this in a future paper.

\section{1. $k$-GRAPHS WITH DEVIATION 1}

In this section we characterize those $k$-graphs $H=H^{(k)}=\left(V, \mu_{H}\right)$ that have $\operatorname{dev} H=1$. These are important since it is precisely the $k$-graphs $G$ with $\operatorname{dev} G<1$ for which $G^{\square t}$ becomes quasi-random as $t \rightarrow \infty$.

To begin our discussion we need to introduce the coboundary operators $\delta^{(i)}$, $i \geq 0$, mapping $k$-graphs $H=\left(V, \mu_{H}\right)$ to $(k+i)$-graphs $\delta^{(i)}(H)=\left(V, \mu_{\delta^{(i)}(H)}\right)$, defined by taking, for $X \in\left(\begin{array}{c}V \\ k+i\end{array}\right)$,

$$
\mu_{\delta^{(i)}(H)}(X)=\prod_{Y \in\left(\begin{array}{l}
X \\
k
\end{array}\right)} \mu_{H}(Y) .
$$


Thus, $X$ is an edge of $\delta^{(i)}(H)$ if and only if $X$ contains an odd number of edges of $H$ as subsets. In particular, $\delta^{(0)}(H)$ is just $H$ itself. (A more general version of this definition occurs in $\mathrm{Hu}$ [H49]). We next establish several basic properties of $\delta^{(i)}$. For integers $a, b \geq 0$, let us say that $a$ and $b$ are disjoint base 2, if the base 2 expansions of $a$ and $b$ have no common 1's. That is, if $a=\sum_{i \geq 0} a_{i} 2^{i}, b=\sum_{i \geq 0} b_{i} 2^{i}, a_{i}, b_{i} \in\{0,1\}$, then $a$ and $b$ are disjoint base 2 if and only if $a_{i} b_{i}=0$ for all $i$.

Also, we let $H_{\varnothing}(k)=\left(V, \mu_{\varnothing}\right)$ denote the trivial $k$-graph on $V$, i.e., with

$$
\mu_{\varnothing}(X)=1 \text { for all } X \in\left(\begin{array}{l}
V \\
k
\end{array}\right) \text {. }
$$

Fact 11.1. For $a, b \geq 0$,

$$
\delta^{(a)}\left(\delta^{(b)}(H)\right)= \begin{cases}\delta^{(a+b)}(H) & \text { if a and b are disjoint base 2 }, \\ H_{\varnothing} & \text { otherwise. }\end{cases}
$$

Proof. For $X \in\left(\begin{array}{c}V \\ k+a+b\end{array}\right)$,

$$
\begin{aligned}
\mu_{\delta^{(a)}\left(\delta^{(b)}(H)\right)}(X) & =\prod_{Y \in\left(\begin{array}{c}
X \\
k+b
\end{array}\right)} \mu_{\delta^{(b)}(H)}(Y) \\
& =\prod_{Y \in\left(\begin{array}{c}
X \\
k+b
\end{array}\right)} \prod_{Z \in\left(\begin{array}{c}
Y \\
k
\end{array}\right)} \mu_{H}(Z)=\prod_{Z \in\left(\begin{array}{c}
X \\
k
\end{array}\right)} \prod_{\substack{C \subset Y \subset X \\
|Y|=k+b}} \mu_{H}(Z) \\
& =\prod_{Z \in\left(\begin{array}{l}
X \\
k
\end{array}\right)} \mu_{H}(Z)^{\left(\begin{array}{c}
a+b \\
a
\end{array}\right)}= \begin{cases}\mu_{\delta^{(a+b)}(H)}(Z) & \text { if }\left(\begin{array}{c}
a+b \\
a
\end{array}\right) \text { is odd }, \\
1 & \text { if }\left(\begin{array}{c}
a+b \\
a
\end{array}\right) \text { is even. }\end{cases}
\end{aligned}
$$

However, $\left(\begin{array}{c}a+b \\ a\end{array}\right)$ is odd if and only if $a$ and $b$ are disjoint base 2 (e.g., see [GKP89]) and the proof is complete.

As an immediate consequence we have $\delta^{(i)} \cdot \delta^{(i)}=0$, the trivial map (sending $H^{(k)}$ to $H_{\varnothing}^{(k+2 i)}$ ) for every $i>0$.

Fact 11.2. For k-graphs $H$ and $H^{\prime}$ on $V$,

$$
\delta^{(i)}\left(H \nabla H^{\prime}\right)=\delta^{(i)}(H) \nabla \delta^{(i)}\left(H^{\prime}\right) .
$$

Proof. For $X \in\left(\begin{array}{c}V \\ k+i\end{array}\right)$,

$$
\begin{aligned}
\mu_{\delta^{(i)}\left(H \nabla H^{\prime}\right)}(X) & =\prod_{Y \in\left(\begin{array}{c}
X \\
k
\end{array}\right)} \mu_{H \nabla H^{\prime}}(Y)=\prod_{Y \in\left(\begin{array}{c}
X \\
k
\end{array}\right)} \mu_{H}(Y) \mu_{H^{\prime}}(Y) \\
& =\prod_{Y \in\left(\begin{array}{l}
X \\
k
\end{array}\right)} \mu_{H}(Y) \prod_{Y \in\left(\begin{array}{l}
X \\
k
\end{array}\right)} \mu_{H^{\prime}}(Y)=\mu_{\delta^{(i)}(H)}(X) \mu_{\delta^{(i)}\left(H^{\prime}\right)}(X) .
\end{aligned}
$$

Thus,

$$
\delta^{(i)}\left(H \nabla H^{\prime}\right)=\delta^{(i)}(H) \nabla \delta^{(i)}\left(H^{\prime}\right) .
$$


We will use the convention that for any set $V$, there are just two distinct 0 -graphs $H^{(0)}=(V, \mu)$. One is $H_{\varnothing}^{(0)}$ for which $\mu \equiv 1$; the other is the “complement" $\bar{H}_{\varnothing}^{(0)}$ for which $\mu \equiv-1$.

The main result of this section is the following.

Theorem 11.1. For a k-graph $H^{(k)}=(V, \mu)$,

$$
\operatorname{dev} H^{(k)}=1
$$

if and only if

$$
H^{(k)}=\nabla_{i=1}^{k} \delta^{(i)}\left(H^{(k-i)}\right)
$$

for some choice of $(k-i)$-graphs $H^{(k-i)}=\left(V, \mu_{k-i}\right), 1 \leq i \leq k$.

Proof. $\Leftarrow$ : Assume $G=G^{(m)}$ and fix $i \geq 1$. By Definition $(\overline{3.1})$,

$$
\operatorname{dev} \delta^{(i)}(G)=\frac{1}{n^{2 m}} \sum_{v} \prod_{\bar{\varepsilon}} \bar{\mu}_{\delta^{(i)}(G)}(\bar{v}(\bar{\varepsilon})) .
$$

We must show that each of the summands is 1 . We use the notation $\bar{u} \in\left(\begin{array}{l}\bar{x} \\ m\end{array}\right)$ for $\bar{x} \in V^{m+i}$ to indicate that $\bar{u}$ is a subsequence of $\bar{x}$ of length $m$. Then

$$
\begin{aligned}
& \prod_{\bar{\varepsilon}} \bar{\mu}_{\delta^{(i)}(G)}(\bar{v}(\bar{\varepsilon}))=\prod_{\bar{\varepsilon}} \prod_{\bar{u} \in\left(\begin{array}{c}
(\bar{\varepsilon}) \\
m
\end{array}\right)} \bar{\mu}_{G}(\bar{u}) \\
& =\prod_{u \in\left(\begin{array}{c}
v(\bar{\varepsilon}) \\
m
\end{array}\right)} \bar{\mu}_{G}(\bar{u})^{2^{i}}=1
\end{aligned}
$$

since for each $\bar{u} \in\left(\begin{array}{c}v(\bar{\varepsilon}) \\ m\end{array}\right)$, there are $i$ unselected coordinates, each of which has 2 (ordered) choices. The proof of (11.4) now follows by repeated application of (4.14).

$\Rightarrow$ : Suppose $H^{(k)}=H^{(k)}(n)=(V, \mu)$ has edge set $E=E\left(H^{(k)}\right)$ and satisfies $\operatorname{dev} H^{(k)}=1$. We proceed by induction on $k$ and then on $n$. For $k=1$ the assertion is immediate since in this case we must have either $E=V$ or $E=\varnothing$. The first case is just $H^{(1)}=\delta^{(1)}\left(\bar{H}_{\varnothing}^{(0)}\right)$; the second is just $H^{(1)}=$ $\delta^{(1)}\left(H_{\varnothing}^{(0)}\right)$. Assume for some $k>1$ that the assertion holds for all values less than $k$. Now, if $n=k$ the only possibility is that $H^{(k)}$ has no edges, i.e., $\mu \equiv 1$. In this case, $H^{(k)}=\delta^{(1)}\left(H_{1}^{(k-1)}\right)$ where $H_{1}^{(k-1)}$ is a $(k-1)$-graph on $V$ having no edges. So, assume the implication holds for all values less than some $n>k$. We will show that it also holds for $n$.

Select an arbitrary fixed vertex $x \in V$ and form $G^{(k)}=H^{(k)} \nabla \delta^{(1)}\left(H^{(k)}(x)\right)$, where $H^{(k)}(x)$ is the neighborhood graph of $H^{(k)}$ at $x$. It is easy to check that $x$ is isolated in $G^{(k)}$, i.e., no edge of $G^{(k)}$ contains $x$. Furthermore, by (4.14)

$$
\operatorname{dev} G^{(k)}=\operatorname{dev}\left(H^{(k)} \nabla \delta^{(1)}\left(H^{(k)}(x)\right)\right)=1
$$


since $\operatorname{dev} H^{(k)}=1$ (by hypothesis) and $\operatorname{dev} \delta^{(1)}\left(H^{(k)}(x)\right)=1$ by the first part of Theorem 11.1 .

Now, define $V^{-}:=V \backslash\{x\}$ and $G^{-}:=G^{(k)}\left[V^{-}\right]$. Thus, $X \subset\left(\begin{array}{l}V \\ k\end{array}\right)$ is an edge of $G$ if and only if $X$ is an edge of $G^{-}$. Consequently, $\operatorname{dev} G^{-}=1$. However, $G^{-}$is a $k$-graph on $n-1$ vertices so by induction we have

$$
G^{-}=\nabla_{i=1}^{k} \delta^{(i)} G^{(k-i)}
$$

for some choice of $(k-i)$-graphs $G^{(k-i)}$ on $V^{-}, 1 \leq i \leq k$. To complete the proof, define $(k-i)$-graphs $H^{(k-i)}$ and $(k-i-1)$-graphs $G_{+}^{(k-i-1)}$ on $V$ by taking

$$
\begin{aligned}
E\left(H^{(k-i)}\right) & =E\left(G^{(k-i)}\right), \\
E\left(G_{+}^{(k-i-1)}\right) & =\left\{X \cup\{x\} \mid X \in G^{(k-i)}\right\}
\end{aligned}
$$

for $1 \leq i \leq k$. The last (straightforward) computation to check is that

$$
H^{(k)}=\nabla_{i=1}^{k}\left(\left(\delta^{(i)}\left(H^{(k-i)}\right) \nabla \delta^{(i-1)}\left(G_{+}^{(k-i-1)}\right)\right)\right.
$$

which then by Fact 11.2 yields the desired representation.

We have normally assumed for $k$-graphs $H=(V, \mu)$ that $\mu=1$ if two arguments are equal. In the case of graphs, this is just the assumption that $H$ has no loops. With this requirement we can assume that the final factor in (11.5) is trivial, i.e., $\delta^{(k)}\left(H_{\varnothing}^{(0)}\right)$. This $k$-graph is just $H_{\varnothing}^{(k)}$ (having no edges) and consequently does not affect the product. Thus, for graphs $G$ we have:

$$
\operatorname{dev} G=1 \Leftrightarrow G \text { is a complete bipartite graph } K_{r, s} \text {. }
$$

Note that if either $r$ or $s$ is 0 , then $G$ just consists of isolated points.

We remark that this section contains the seeds from which various cohomological aspects of $k$-graphs can be developed. We have begun this in [CG(b)].

\section{SOME EXPLICIT CONSTRUCTIONS}

In this section we give a few of the simplest constructions for $k$-graphs having small deviation or $l$-deviation. By the earlier results, these $k$-graphs consequently behave like random $k$-graphs in many respects, and can often be used in place of random $k$-graphs. Unlike random $k$-graphs, however, their precise structure is determined, and can be employed in other ways. A typical example of this phenomenon is the case of so-called "expander" graphs in communication networks. Random graphs have excellent expanding properties but are difficult to use when precise algorithms for routing (for example) are required. To begin with, suppose $H_{i}, 1 \leq i \leq m$, is a family of $k$-graphs. By (4.8) we have for $H^{*}:=\square_{i=1}^{m} H_{i}$,

$$
\operatorname{dev} H^{*}=\prod_{i=1}^{m} \operatorname{dev} H_{i}
$$


Thus, if each $\operatorname{dev} H_{i}$ is bounded away from 1, then $\operatorname{dev} H^{*}=o(1)$ as $m \rightarrow \infty$. In particular, if we take all $H_{i}$ equal to a fixed $k$-graph $H$ with $\operatorname{dev} H=c<1$ then

$$
\operatorname{dev} H^{\square m}=c^{m},
$$

from which many quantitative quasi-random properties easily follow.

A more arithmetic family of $k$-graphs with small deviation is given by the following construction. Let $p$ denote a fixed (arbitrary) prime. Form the "Paley" $k$-graph $P_{p}^{(k)}=\left(V_{p}, \mu_{p}\right)$ as follows:

$V_{p}:=G F(p)$, the finite field with $p$ elements.

For $X \in\left(\begin{array}{c}V_{p} \\ k\end{array}\right), \mu_{p}(X):=\phi\left(\sum_{x \in X} x(\bmod p)\right)$ where $\phi: G F(p) \rightarrow$

$\{1,-1\}$ denotes the nonprincipal quadratic character on $G F(p)$.

Thus, $\left\{v_{1}, \ldots, v_{k}\right\}$ is an edge of $P^{(k)}(p)$ iff $v_{1}+\cdots+v_{k}$ is a quadratic nonresidue in $G F(p)$.

\section{Fact 12.1.}

$$
\operatorname{dev} P_{p}^{(k)}=O\left(p^{-1}\right), \quad p \rightarrow \infty .
$$

Proof. We will use the following well-known estimate of Burgess [B62] (also see Weil [We48]). For distinct $a_{1}, \ldots, a_{s} \in G F(p)$,

$$
\left|\sum_{x \in G F(p)} \phi\left(x+a_{1}\right) \cdots \phi\left(x+a_{s}\right)\right| \leq(s-1) \sqrt{p} .
$$

Note that (12.1) holds for nondistinct $a_{i}$ as well, provided the product is not identically one. Then

$$
\begin{aligned}
& p^{2 k} \operatorname{dev} P_{p}^{(k)}=\sum_{\substack{x_{i}(0), x_{i}(1) \\
1 \leq i \leq k}} \prod_{\substack{\varepsilon \in\{0,1\} \\
1 \leq j \leq k}} \bar{\mu}_{p}\left(x_{1}\left(\varepsilon_{1}\right), \ldots, x_{k}\left(\varepsilon_{k}\right)\right) \\
& =\sum_{\substack{x_{i}(0), x_{i}(1) \\
1 \leq i \leq k}} \prod_{\substack{\varepsilon_{j} \in\{0,1\} \\
1 \leq j \leq k}} \phi\left(x_{1}\left(\varepsilon_{1}\right)+\cdots+x_{k}\left(\varepsilon_{k}\right)\right) \\
& =\sum_{\substack{x_{i}(0), x_{i}(1) \\
2 \leq i \leq k}}\left(\sum_{x} \prod_{\substack{\varepsilon_{j} \\
2 \leq j \leq k}} \phi\left(x+x_{2}\left(\varepsilon_{2}\right)+\cdots+x_{k}\left(\varepsilon_{k}\right)\right)\right)^{2} \\
& \leq \sum_{\substack{x_{i}(0), x_{i}(1) \\
2 \leq i \leq k}}^{\prime}\left(2^{k-1} \sqrt{p}\right)^{2}+\sum_{\substack{x_{i}(0), x_{i}(1) \\
2 \leq i \leq k}}^{\prime \prime} p^{2} \text { by (12.2) }
\end{aligned}
$$

where $\Sigma^{\prime}$ denotes the sum over all choices of the $x_{i}\left(\varepsilon_{i}\right)$ for which some value $v \in G F(p)$ occurs as a sum $x_{2}\left(\varepsilon_{2}\right)+\cdots+x_{k}\left(\varepsilon_{k}\right)$ in an odd number of ways (and $\Sigma^{\prime \prime}$ represents the complementary set). Consequently,

$$
p^{2 k} \operatorname{dev} P_{p}^{(k)}=O\left(p^{2 k-1}\right), \quad p \rightarrow \infty .
$$

This implies (12.1) and Fact 12.1 is proved. 
We point out that essentially the same arguments (from [C90]; see also [GS71]) show that for $1 \leq l<k$, the $k$-graph $G_{l}=\delta^{(k-l)}\left(P_{p}^{(l)}\right)$ satisfies

$$
\operatorname{dev}_{l} G_{l}=o(1), \quad \operatorname{dev}_{l+1} G_{l} \geq(1+o(1)) 2^{-\left(\begin{array}{l}
k \\
l
\end{array}\right), \quad p \rightarrow \infty .}
$$

It would be interesting to know if in fact we could have

$$
\operatorname{dev}_{l} G_{l}^{\prime}=o(1), \quad \operatorname{dev}_{l+1} G_{l}^{\prime}=1+o(1), \quad n \rightarrow \infty
$$

for a suitable family of $k$-graphs $G_{l}^{\prime}$ on $n$ vertices.

We will describe one more class of quasi-random families of $k$-graphs, the so-called "even intersection" $k$-graphs $I^{(k)}(n)$. The vertex set of $I^{(k)}(n)$ is $2^{[n]}$, the collection of all subsets of $\{1,2, \ldots, n\}$. A $k$-set $\left\{X_{1}, \ldots, X_{k}\right\}$, $X_{i} \in 2^{[n]}$, is an edge of $I^{(k)}(n)$ if and only if

$$
\left|\bigcap_{i=1}^{k} X_{i}\right| \equiv 0 \quad(\bmod 2) .
$$

Let $\mu$ denote the (multiplicative) edge function for $I^{(k)}(n)$.

\section{Fact 12.2.}

Proof. Define $N:=2^{n}$. Since

$$
\operatorname{dev} I^{(k)}(n)=o(1), \quad n \rightarrow \infty .
$$

$$
\begin{aligned}
\operatorname{dev} I^{(k)}(n) & =\frac{1}{N^{2 k}} \sum_{\substack{X_{i}(0), X_{i}(1) \\
1 \leq i \leq k}} \prod_{\substack{\varepsilon_{j} \in\{0,1\} \\
1 \leq j \leq k}} \bar{\mu}\left(X_{1}\left(\varepsilon_{1}\right), \ldots, X_{k}\left(\varepsilon_{k}\right)\right) \\
& =\frac{1}{N^{2 k}} \sum_{\substack{X_{i}(0), X_{i}(1) \\
2 \leq i \leq k}}\left(\sum_{X} \prod_{\substack{\varepsilon_{j} \\
2 \leq i \leq k}} \bar{\mu}\left(X, X_{2}\left(\varepsilon_{2}\right), \ldots, X_{k}\left(\varepsilon_{k}\right)\right)\right)^{2} .
\end{aligned}
$$

then it suffices to show that the number of $X \subset[n]$ for which the sum

$$
S:=\sum_{\varepsilon_{2}, \ldots, \varepsilon_{k}}\left|X \cap X_{2}\left(\varepsilon_{2}\right) \cap \cdots \cap X_{k}\left(\varepsilon_{k}\right)\right|
$$

is even is $(1+o(1)) N / 2$ for almost all choices of $X_{i}(0), X_{i}(1) \subset[n], 2 \leq i \leq k$.

We will show that this is in fact the case whenever all the $X_{i}(0)$ and $X_{i}(1)$ are distinct. First note that the parity of $S$ is unchanged if we make the replacements:

$$
\begin{aligned}
& X_{i}(0) \rightarrow X_{i}^{\prime}(0):=X_{i}(0) \backslash X_{i}(1), \\
& X_{i}(1) \rightarrow X_{i}^{\prime}(1):=X_{i}(1) \backslash X_{i}(0)
\end{aligned}
$$

since each element $x \in X_{i}(0) \cap X_{i}(1)$ affects an even number of terms of $S$. By construction, $X_{i}^{\prime}(0)$ and $X_{i}^{\prime}(1)$ are disjoint. 
Thus, we have reduced our problem to counting the number of $X \subset[n]$ for which the sum

$$
S^{\prime}:=\sum_{\varepsilon_{2}, \ldots, \varepsilon_{k}}\left|X \cap X_{2}^{\prime}\left(\varepsilon_{2}\right) \cap \cdots \cap X_{k}^{\prime}\left(\varepsilon_{k}\right)\right|
$$

is even. Let

$$
s\left(\varepsilon_{2}, \ldots, \varepsilon_{k}\right):=s(\bar{\varepsilon}):=\left|X_{2}^{\prime}\left(\varepsilon_{2}\right) \cap \cdots \cap X_{k}^{\prime}\left(\varepsilon_{k}\right)\right| .
$$

Since all $2^{k-1}$ expressions on the right-hand side of (12.3) are disjoint, then the number of $X$ such that $S$ is even is just

$$
\sum_{i(\bar{\varepsilon})}^{\prime} \prod_{\bar{\varepsilon}}\left(\begin{array}{c}
s(\bar{\varepsilon}) \\
i(\bar{\varepsilon})
\end{array}\right) 2^{n-s}
$$

where the sum $\sum^{\prime}$ is taken over all $i(\bar{\varepsilon})$ such that $\sum_{\bar{\varepsilon}} i(\bar{\varepsilon}) \equiv 0(\bmod 2)$, and $\bar{\varepsilon}:=\left(\varepsilon_{2}, \ldots, \varepsilon_{k}\right), s:=\sum_{\bar{\varepsilon}} s(\bar{\varepsilon})$. The interpretation of (12.4) is simply that $S^{\prime}$ counts the number of ways of choosing $X$ which has $i(\bar{\varepsilon})$ elements $X_{2}^{\prime}\left(\varepsilon_{2}\right) \cap \cdots \cap X_{k}^{\prime}\left(\varepsilon_{k}\right)$. Of course, $S^{\prime}$ is not affected if $X$ is changed by any subset of $[n] \backslash \bigcup_{i, j} X_{i}^{\prime}\left(\varepsilon_{j}\right)$; this accounts for the factor $2^{n-s}$ in (12.4).

However, observe that

$$
\sum_{i(\bar{\varepsilon})}(-1)^{\sum_{\bar{\varepsilon}} i(\bar{\varepsilon})} \prod_{\bar{\varepsilon}}\left(\begin{array}{c}
s(\bar{\varepsilon}) \\
i(\bar{\varepsilon})
\end{array}\right) 2^{n-s}=0
$$

since this is just the result of expanding the expression

$$
\prod_{\bar{\varepsilon}}(x-1)^{s(\bar{\varepsilon})}
$$

and substituting $x=1$. Thus, the expression in (12.5) summed over $i(\bar{\varepsilon})$ with $\sum_{\bar{\varepsilon}} i(\bar{\varepsilon})$ even is just one-half of the total sum

$$
\sum_{i(\bar{\varepsilon})} \prod_{\bar{\varepsilon}}\left(\begin{array}{c}
s(\bar{\varepsilon}) \\
i(\bar{\varepsilon})
\end{array}\right) 2^{n-s}=2^{n}
$$

i.e., $2^{n-1}$, which is $N / 2$, as required. Since almost all choices of the $X_{i}\left(\varepsilon_{j}\right)$ result in distinct sets then Fact 12.2 is proved.

We remark that the same techniques can be applied to a variety of other families of subsets formed by modular restrictions on intersections, e.g., such as the $k$-graph having vertex set $\left(\begin{array}{c}{[2 n]} \\ n\end{array}\right)$ and edges $\left\{X_{1}, \ldots, X_{k}\right\}, X_{i} \in\left(\begin{array}{c}{[2 n]} \\ n\end{array}\right)$, with $\left|X_{1} \cap \cdots \cap X_{k}\right| \equiv 0(\bmod 2)$.

\section{Concluding REMARKS}

In a series of papers, Thomason [T87(a), T87(b), T89] and HavilandThomson [H89, HT89, HT(a)] have investigated a concept called " $(p, \alpha)$ jumbledness," which is related to our work on quasi-randomness. Restricting 
the discussion to the case $p=1 / 2$ (which is our primary focus), a $k$-graph $H$ with vertex set $V$, is said to be $\left(\frac{1}{2}, \alpha\right)$-jumbled provided

$$
\left|e(H[X])-\frac{1}{2}\left(\begin{array}{c}
|X| \\
k
\end{array}\right)\right| \leq \alpha|X|
$$

for all $X \subset V$. Here, $\alpha$ is ordinarily some function of $n=|V|$. It turns out that for $k=2$, the condition that $\alpha=o(n)$ is precisely a quasi-random property of graphs (and so, is equivalent to $\operatorname{dev} H=o(1), n \rightarrow \infty$ ). However, for $k>2$, this property is considerably weaker than being quasi-random. More precisely, it is equivalent to $\operatorname{dev}_{2} H=o(1)$ (see $\S 8$ and [C90]), whereas quasirandomness of a $k$-graph is equivalent to $\operatorname{dev}_{k} H=o(1)$. We remark that almost all random 2-graphs $G_{1 / 2}$ on a vertex set $V$ have been shown by Spencer [S89] to have the following property: For $H=\delta^{(1)}\left(G_{1 / 2}\right)$, the 1-coboundary of $G_{1 / 2}($ see $\S 11)$, we have

$$
\left|e(H[X])-\frac{1}{2}\left(\begin{array}{c}
|X| \\
3
\end{array}\right)\right| \leq 200|X|^{2}
$$

for all $X \subset V$. However, $\operatorname{dev} H=1$, and, in particular $H$ contains no induced 4-vertex subgraph with an odd number of edges. Thus, while $(p, \alpha)$ jumbledness is an effective concept for studying random behavior in graphs, it appears to be too weak to carry out the analogous investigations for general $k$-graphs.

In the same spirit as (13.1), it is not hard to prove the following bound for any $k$-graph $H$ on an $n$-vertex set $V:$ For any $X \subset V$ with $x:=|X|$, we have

$$
\left|x^{k}-2 k ! e(H[X])\right| \leq\left(\left(\frac{n}{x}\right)^{2 k} \operatorname{dev} H\right)^{2^{-k}} x^{k}
$$

No doubt, the exponent $2^{-k}$ here can be improved, as can many of the other constants in our various estimates. We have no idea what the truth should be.

It would be most interesting to know other quasi-random properties of $k$ graphs. In the case of graphs, Simonovits and Sós [SS91] have very recently proved the following result. We first need several definitions. Let $G$ be a graph with vertex set $V$. For disjoint sets $X, Y \subset V$, let $d(X, Y):=e(X, Y) /|X||Y|$ where $e(X, Y)$ denotes the number of edges with endpoints in both $X$ and $Y$. A pair $(X, Y)$ is called $\varepsilon$-regular if for every $X^{\prime} \subset X, Y^{\prime} \subset Y$ satisfying $\left|X^{\prime}\right|>\varepsilon|X|,\left|Y^{\prime}\right|>\varepsilon|Y|$, we have

$$
\left|d\left(X^{\prime}, Y^{\prime}\right)-d(X, Y)\right|<\varepsilon
$$

A fundamental result of Szemerédi [Sz78] is his

Regularity Lemma. For every $\varepsilon>0$ and $m$, there exists $k(\varepsilon, m)$ such that for every $G(n)$, the vertex set $V(n)$ of $G(n)$ can be partitioned into $k+1$ sets 
$U_{0}, U_{1}, \ldots, U_{k}$ for some $k$ with $m<k<k(\varepsilon, m)$ so that $\left|U_{0}\right|<\varepsilon n$, all $\left|U_{i}\right|$ are equal for $i>0$, and for all except $\varepsilon\left(\begin{array}{l}k \\ 2\end{array}\right)$ pairs $(i, j),\left(U_{i}, U_{j}\right)$ is $\varepsilon$-regular.

It asserts that in a certain sense, any graph can be approximated by a random $k$-partite graph. In [SS91] it is shown that the following property is a quasirandom property.

$\left(P_{S}\right):$ For every $\varepsilon>0$ and $m$, there exist two integers $k(\varepsilon, m)$ and $n_{0}(\varepsilon, m)$ such that for $n>n_{0}, G(n)$ has a "Szemerédi" partition for the parameters $\varepsilon$ and $m$ into $k$ almost equal classes $U_{1}, \ldots, U_{k}$, with $m<k<$ $k(\varepsilon, m)$ so that $\left(U_{i}, U_{j}\right)$ is $\varepsilon$-regular, and $\left|d\left(U_{i}, U_{j}\right)-\frac{1}{2}\right|<\varepsilon$ holds for all except $\varepsilon\left(\begin{array}{l}k \\ 2\end{array}\right)$ pairs $(i, j), 1 \leq i, j \leq k$.

In fact, when a family $\{G(n)\}$ is quasi-random then it is true [SS91] that Szemerédi partitions always exist having no exceptional pairs. The corresponding result for $k$-graphs is given in [C91].

As mentioned at the beginning, most of the preceding analysis can be carried out assuming that the random $k$-graph properties we are trying to classify arise from random $k$-graphs in which $k$-sets are selected with a fixed probability $p \in(0,1)$, rather than probability $1 / 2$. The corresponding statements and arguments are essentially the same although notationally slightly more cumbersome. However, if we allow $p=p(n)$ to depend on $n$, the size of the $k$-graph, then the situation becomes much more complex, especially as $p(n)$ becomes small, e.g., $p(n)=O\left(n^{1 / 2}\right)$. We certainly do not yet have a full understanding of quasi-randomness in this range.

Another direction that merits attention is what we called "forcing families" (for graphs) in [CGW89]. Let us call a family $\mathscr{F}$ of $k$-graphs forcing if whenever $\#\{F<G(n)\}=(1+o(1)) n^{v} 2^{-e}$ for all $F=F(v, e) \in \mathscr{F}$ (i.e., $F$ has $v$ vertices and $e$ edges) then $\{G(n)\}$ is quasi-random. For example, in the case of graphs, it is shown in [CGW89] that the following families are forcing (where $K_{m}$ denotes the complete graph on $m$ vertices, $C_{m}$ denotes the cycle on $m$ vertices and $K_{r, s}$ denotes the complete bipartite graph on $r$ and $s$ vertices):

(i) $\left\{K_{2}, C_{4}\right\}$;

(ii) $\left\{K_{2}, C_{2 t}\right\}$, any fixed $t$;

(iii) $\left\{C_{2 r}, C_{2 s}\right\}, r \neq s$;

(iv) $\left\{K_{2}, K_{2, t}\right\}, t \geq 2$

(v) $\left\{K_{2, s}, K_{2, t}\right\}, s, t \geq 2, s \neq t$.

Can forcing families of graphs be characterized? What is the situation for $k$ graphs?

More generally, one can attempt the same type of classification of random behavior for a wide variety of objects, for example, ordered $k$-graphs, integer sequences, matrices, partially ordered sets, permutations, groups, and vector spaces, to name a few, as well as for functions defined on these and other structures. Preliminary work on some of these topics has recently been initiated (e.g., see [CG(a), CG90(b), CG(c), SS(a), ST(a)]) but clearly a vast expanse of fertile ground still awaits exploration. 


\section{REFERENCES}

[BNS89] L. Babai, N. Nisan, and M. Szégedy, Multi-party protocols and logspace-hard pseudorandom sequences, Proc. 21st Sympos. Theory of Computing, ACM, 1989, pp. 1-11.

[Be89] C. Berge, Hypergraphs, North-Holland, Amsterdam, 1989.

[Bo85] B. Bollobás, Random graphs, Academic Press, New York, 1985.

[Bo79] _ Graph theory, Springer-Verlag, New York, 1979.

[BT81] B. Bollobás and A. Thomason, Graphs which contain all small graphs, European J. Combin. 2 (1981), 13-15.

[BM76] J. A. Bondy and U. S. R. Murty, Graph theory with applications, Elsevier, New York, 1976.

[B62] D. A. Burgess, On character sums and primitive roots, Proc. London Math. Soc. 12 (1962), 179-192.

[C89] F. R. K. Chung, Diameters and eigenvalues, J. Amer. Math. Soc. 2 (1989), 187-196.

[C90] _ Quasi-random classes for hypergraphs, Random Structures and Algorithms 1 (1990), 363-382.

[C91] _ Regularity lemmas for hypergraphs and quasi-randomness, Random Structures and Algorithms 2 (1991) (to appear).

[CG90(a)] F. R. K. Chung and R. L. Graham, Quasi-random hypergraphs, Random Structures and Algorithms 1 (1990), 105-124.

[CG90(b)] __, On graphs with missing prescribed induced subgraphs, A Tribute to Paul Erdös (A. Baker et al., eds.), Cambridge Univ. Press, 1990, pp. 111-120.

[CG(a)] - Maximum cuts and quasi-random graphs (to appear).

$[\mathrm{CG}(\mathrm{b})]-$ Cohomological aspects of hypergraphs, Trans. Amer. Math. Soc. (to appear).

[CG(c)] - Quasi-random tournaments (to appear).

[CGW88] F. R. K. Chung, R. L. Graham, and R. M. Wilson, Quasi-random graphs, Proc. Nat. Acad. U.S.A. 85 (1988), 969-970.

[CGW89] _- Quasi-random graphs, Combinatorica 9 (1989), 345-362.

[CDGT88] D. Cvetković, M. Doob, I. Gutman, and A. Torǵasev, Recent results in the theory of graph spectra, Ann. Discrete Math., no. 36, North-Holland, Amsterdam, 1988.

[CDS80] D. Cvetković, M. Doob, and H. Sacks, Spectra of graphs, Academic Press, New York, 1980.

[ES72] P. Erdös and J. Spencer, Imbalances in k-colorations, Networks 1 (1972), 379-385.

[ES74] — Probabilistic methods in combinatorics, Akadémiai Kiadó, Budapest, 1974.

[ES82] P. Erdös and V. T. Sós, On Ramsey-Turàn type theorems for hypergraphs, Combinatorica 2 (1982), 289-295.

[F76] R. Fagin, Probabilistic on finite models, J. Symbolic Logic 41 (1976), 51-58.

[FR89] P. Frankl and V. Rödl, personal communication.

[FR88] $\quad-$ Some Ramsey-Turán type results for hypergraphs, Combinatorica 8 (1988), 323332.

[FRW88] P. Frankl, V. Rödl, and R. M. Wilson, The number of submatrices of given type in a Hadamard matrix and related results, J. Combin. Theory Ser. B 44 (1988), 317-328.

[FK81] Z. Füredi and J. Komlós, The eigenvalues of random symmetric matrices, Combinatorica 1 (1981), 233-241.

[GKLT69] Y. V. Glebskii, D. I. Kogan, I. M. Liogonki, and V. A. Talanov, The extent and degree of satisfiability of a form of the restricted predicate calculus, Kibernetika 2 (1969), 31-42.

[GKP89] R. L. Graham, D. E. Knuth, and O. Patashnik, Concrete mathematics, Addison-Wesley, Reading, MA, 1989.

[GS71] R. L. Graham and J. H. Spencer, A constructive solution to a tournament problem, Canad. Math. Bull. 14 (1971), 45-48. 
[H89] J. Haviland, Cliques and independent sets, Ph.D. thesis, Cambridge Univ., 1989.

[HT89] J. Haviland and A. Thomason, Pseudo-random hypergraphs, Discrete Math. 75 (1989), 255-278.

[HT(a)] _ Note on testing the 'pseudo-randomness' of a hypergraph (to appear).

[H49] S. T. Hu, A cohomology theory with higher coboundary operators. I, Nederl. Akad. Wetensch. Proc. 52 (1949), 1144-1150.

[L89] L. Lovaśz, personal communication.

[M68] J. Moon, Topics on tournaments, Holt, Rinehart, and Winston, New York, 1968.

[R86] V. Rödl, On the universality of graphs with uniformly distributed edges, Discrete Math. 59 (1986), 125-134.

[S48] C. Shannon, A mathematical theory of communication, Bell System Tech. J. 27 (1948), 379-423.

[SS88] S. Shelah and J. Spencer, Zero-one laws for sparse random graphs, J. Amer. Math. Soc. 1 (1988), 97-106.

[SS91] M. Simonovits and V. T. Sós, Szemerédi partitions and quasi-randomness, Random Structures and Algorithms 2 (1991), 1-10.

[S89] J. Spencer, personal communication.

[ST(a)] J. Spencer and P. Tetali, Quasi-random graphs with tolerance $\varepsilon$, preprint.

[Sz78] E. Szemerédi, On regular partitions of graphs, Problèmes Combinatoire et Théorie des Graphes (J. Bermond et al., eds.) CNRS, Paris, 1978, pp. 399-401.

[T87(a)] A. Thomason, Random graphs, strongly regular graphs and pseudo-random graphs, Surveys in Combinatorics 1987 (C. Whitehead, ed.), London Math. Soc. Lecture Notes Ser., no. 123, Cambridge Univ. Press, Cambridge, 1987, pp. 173-196.

[T87(b)] _ Pseudo-random graphs, Proc. Random Graphs, Poznań 1985 (M. Karonski, ed.), Ann. Discrete Math., no. 33, North-Holland, 1987, pp. 307-331.

[T89] _ Dense expanders and pseudo-random bipartite graphs, Discrete Math. 75 (1989), 381-386.

[Wa88] W. D. Wallis, Combinatorial designs, Marcel Dekker, New York, 1988 (Example 8.5.5 on graphical Hadamard matrices, p. 160).

[We48] A. Weil, Sur les courbes algébrique et les variétés qui séen déduisent, Actualités Sci. Indust., no. 1041, Hermann, Paris, 1948.

[Wi72] R. M. Wilson, Cyclotomy and difference families on abelian groups, J. Number Theory 4 (1972), 17-47.

[Wi74] _- Constructions and uses of pairwise balanced designs, Combinatorics (M. Hall, Jr. and J. H. van Lint, eds.), Math. Centre Tracts, no. 55, Amsterdam, 1974, pp. 18-41.

[Wi90] _ A diagonal form for the incidence matrices of $t$-subsets $v$ s. $k$-subsets, European $\mathrm{J}$. Combin. 11 (1990), 609-615.

Bell Communications Research, Morristown, New Jersey 07960

aT\&T Bell laboratories, Murray Hill, New Jersey 07974 